\title{
A rational moment problem on the unit circle*
}

\author{
A. Bultheel’ P. González-Vera
}

\begin{abstract}
Let $\left\{\alpha_{k}\right\}_{k=1}^{\infty}$ be a sequence of not necessarily distinct points on the complex unit circle. We consider the moment problem where it is to find a positive measure on $[-\pi, \pi]$ such that for $\omega_{0}=1$ and $\omega_{n}(z)=\left(z-\alpha_{1}\right) \cdots\left(z-\alpha_{n}\right), n=1,2, \ldots$ we have

$$
\int_{-\pi}^{\pi} d \mu(\theta)=1, \quad \int_{-\pi}^{\pi} \frac{d \mu(\theta)}{\omega_{n}\left(e^{i \theta}\right)}=\mu_{n}, \quad n=1,2, \ldots
$$

for a given sequence of moments $\left\{\mu_{n}\right\}_{n=0}^{\infty}$. This paper gives results which to some extend generalise the limit point - limit circle situation of classical moment problems.
\end{abstract}

Keywords: general moment problem, orthogonal rational functions, nested disks.

AMS Classification: 30E05, 42C10, 42A99, 47A57

\section{Introduction}

Let $\mathbb{T}=\{z \in \mathbb{C}:|z|=1\}$ be the complex unit circle and let $\left\{\alpha_{k}\right\}_{k=1}^{\infty}$ be a sequence of not necessarily distinct points on $\mathbb{T} \backslash\{1\}$. Introduction of the "forbidden" point 1 is not a severe restriction because there is only a countable number of $\alpha_{k}$ 's so that there always exists such a point on $\mathbb{T}$, which by a simple rotation can be brought to the position 1 . Define $\omega_{n}(z)=\prod_{k=1}^{n}\left(z-\alpha_{k}\right)$ for $n \geq 1$ and set $\omega_{0}=1$. By $\Pi_{n}$ we denote the set of polynomials of degree at most $n$. We consider the spaces

$$
\mathcal{L}_{n}=\left\{\frac{p_{n}(z)}{\omega_{n}(z)}: p_{n} \in \Pi_{n}\right\}, \quad \mathcal{L}=\cup_{k=0}^{\infty} \mathcal{L}_{k}
$$

For any complex function $f$, let $f_{*}(z)=\overline{f(1 / \bar{z})}$. It is obvious that $\mathcal{L}_{n *}=\left\{f: f_{*} \in \mathcal{L}_{n}\right\}=\mathcal{L}_{n}$ and similarly $\mathcal{L}_{*}=\mathcal{L}$. Finally, we set $\mathcal{R}_{n}=\mathcal{L}_{n} \cdot \mathcal{L}_{n}$ and $\mathcal{R}=\mathcal{L} \cdot \mathcal{L}$. Let $M$ be a linear functional defined on $\mathcal{L} \cdot \mathcal{L}$ which is real and positive, i.e., which satisfies

$$
M\left\{f_{*}\right\}=\overline{M\{f\}}, \quad f \in \mathcal{L} \cdot \mathcal{L} \quad \text { and } \quad M\left\{f f_{*}\right\}>0, \quad 0 \neq f \in \mathcal{L} .
$$

This functional defines an inner product by

$$
\langle f, g\rangle=M\left\{f g_{*}\right\}
$$

*This research was performed as part of the European project RoLLS under contract CHRX-CT93-0416.

${ }^{\dagger}$ Department of Computer Science, K.U.Leuven, Belgium

${ }^{\ddagger}$ Department Análisis Math., Univ. La Laguna, Tenerife, Spain

$\S$ Department of Math., Univ. of Amsterdam, The Netherlands

IDepartment of Math. Sciences, Norwegian Univ. of Science and Technology, Trondheim, Norway 
An example of such a linear functional is given by

$$
M\{f\}=\int_{-\pi}^{\pi} f\left(e^{i \theta}\right) d \mu(\theta), \quad\langle f, g\rangle=\int_{-\pi}^{\pi} f\left(e^{i \theta}\right) \overline{g\left(e^{i \theta}\right)} d \mu(\theta)
$$

where $\mu$ is a positive measure on $[-\pi, \pi]$. The subject of this paper, just as in Section 8 of [3], is to solve the following moment problem: Given the moments $\left\{\mu_{n}\right\}_{n=0}^{\infty}$, defined by

$$
M\{1\}=\mu_{0} \quad \text { and } \quad M\left\{\frac{1}{\omega_{n}}\right\}=\mu_{n}, \quad n=1,2, \ldots
$$

(without loss of generality we may assume that $\mu_{0}=1$ ), does there exist a finite and positive measure $\mu$ on $[-\pi, \pi]$ such that

$$
M\left\{\frac{1}{\omega_{n}}\right\}=\mu_{n}=\int_{-\pi}^{\pi} \frac{d \mu(\theta)}{\omega_{n}\left(e^{i \theta}\right)}, \quad n=0,1, \ldots
$$

It was shown in [3] that this moment problem always has at least one solution. This existence result was proved in a constructive way. Such a solution was obtained as the limit of a converging subsequence of quadrature formulas. Since there could be different subsequences, converging to different limits, there might be (infinitely) many solutions. Here we will address the problem whether the moment problem is determinate or indeterminate, i.e., whether a solution $\mu$ is unique or not. We shall, to a certain extend, generalise the classical limiting point versus limiting disk situation for a sequence of nested disks. This technique was known to Weyl [23] and used by Akhiezer [1] and Shohat and Tamarkin [20] when they studied the classical Hamburger moment problem to characterize the situation where the moment problem has a unique solution. See also the work of Stone [22].

For more information on moment problems and nested disks see [10, 11, 12, 21, 14]. Uniqueness criteria for the strong Hamburger moment problem can be found in [17]. An extended Hamburger moment problem was discussed in [15]. In the Hamburger problem, the unit circle is replaced by the real line. Similar results about unique solvability were obtained in [16] for the extended Hamburger moment problem where a finite number of points $\alpha_{k}$ on the real line are cyclically repeated. Multipoint matrix versions of the Hamburger and Stieltjes moment problems are found in e.g., $[19,18]$. In the case where the points $\alpha_{k}$ are located inside the unit disk, results similar to the ones of this paper were given in [7] and [4]. Here we treat the case of the unit circle and at the same time we consider a general sequence of $\alpha_{k}$ 's which need not be cyclically repeated. We emphasize that the theory where all the points $\alpha_{k}$ lie inside the open unit disk is substantially different from the theory where they are on its boundary. In the boundary case, nontrivial new problems arise e.g. due to the fact that functions in $\mathcal{L}$ are not continuous on the unit circle anymore. Analog results for the real line can be obtained in a similar way. However, there the problem is that the support of the measure is not compact and special attention has to be paid to the point at infinity which causes some trouble. For simplicity, we discuss here only the case of the unit circle. An essential role will be played by the orthogonal rational functions. They play the role of orthogonal polynomials in the Hamburger case or the orthogonal Laurent polynomials [13] in the case of the strong Hamburger moment problem. For the case of points inside the unit disk, such orthogonal rational functions were first studied in $[8,2,9]$. For points on the boundary, they appear in $[16,3,6,5]$ 


\section{Orthogonal rational functions and recurrence rela- tion}

First we observe that

$$
\mathcal{L}_{n}=\operatorname{span}\left\{b_{0}, b_{1}, \ldots, b_{n}\right\}
$$

with

$$
b_{0}=1, \quad b_{n}=Z_{1} Z_{2} \cdots Z_{n}, \quad n \geq 1
$$

where

$$
Z_{k}(z)=\frac{i(z-1)\left(\alpha_{k}-1\right)}{z-\alpha_{k}}, \quad k \geq 1
$$

We use this notation also for $k=0$, in which case we set $\alpha_{0}=-1$. Thus

$$
Z_{0}(z)=2 i \frac{1-z}{1+z}
$$

Note that the basis functions $b_{k}$ satisfy $b_{k *}=b_{k}$. By a Gram-Schmidt procedure, these basis functions are orthogonalised to give the orthonormal functions $\phi_{n}, n=0,1, \ldots$ Let

$$
\phi_{n}(z)=\phi_{n}(1)+\cdots+\kappa_{n}^{\prime} b_{n-1}(z)+\kappa_{n} b_{n}(z) .
$$

The orthonormal functions can be fixed uniquely by requiring $\kappa_{n}>0$. This is what will always be assumed when we refer to the orthonormal functions. Note that then, because of $M\{1\}=1$ we find $\phi_{0}=\kappa_{0}=1$. Furthermore

$$
\kappa_{n}=\left[\frac{\phi_{n}(z)}{b_{n}(z)}\right]_{z=\alpha_{n}} \quad \text { and } \quad \kappa_{n}+\frac{\kappa_{n}^{\prime}}{Z_{n}\left(\alpha_{n-1}\right)}=\left[\frac{\phi_{n}(z)}{b_{n}(z)}\right]_{z=\alpha_{n-1}}, n \geq 1 .
$$

If we set $\phi_{n}=p_{n} / \omega_{n}$, with $p_{n} \in \Pi_{n}$, then we say that $\phi_{n}$ (and also its index $n$ ) is singular if $p_{n}\left(\alpha_{n-1}\right)=0$. Otherwise, they are called regular. The system $\left\{\phi_{n}\right\}$ is called regular if all the indices are regular.

With this normalization we have

Lemma 2.1 The orthonormal functions $\phi_{n}$ have real coefficients with respect to the basis $b_{k}$ and $\phi_{n *}=\phi_{n}$.

Proof. Because $b_{k *}=b_{k}$, it is obvious that if the coefficients are real, also $\phi_{n *}=\phi_{n}$. The proof of real coefficients follows easily by induction. The result is true for $n=0$. Suppose it is true for $i \leq n-1$, then by the Gram-Schmidt procedure

$$
\phi_{n}=\chi_{n} /\left\|\chi_{n}\right\|, \quad \text { with } \quad \chi_{n}=b_{n}-\sum_{i=0}^{n-1} \gamma_{i} \phi_{i}, \quad \gamma_{i}=\left\langle b_{n}, \phi_{i}\right\rangle .
$$

Using $M\left\{f_{*}\right\}=\overline{M\{f\}},\langle f, g\rangle=M\left\{f g_{*}\right\}, b_{n *}=b_{n}$ and $\phi_{i *}=\phi_{i}$ for $i<n$, it follows that the coefficients $\gamma_{i}=\left\langle b_{n}, \phi_{i}\right\rangle=M\left\{b_{n} \phi_{i *}\right\}=\overline{M\left\{b_{n *} \phi_{i}\right\}}=\overline{M\left\{b_{n} \phi_{i *}\right\}}=\bar{\gamma}_{i}$ are real. Since $\phi_{i}$ has real coefficients with respect to the basis $b_{k}$, also $\chi_{n}$ and thus also $\phi_{n}$ will have real coefficients with respect to the basis $b_{k}$.

The following is a slight adaptation of Theorem 4.1 in [3]. 
Theorem 2.2 Suppose the system $\left\{\phi_{n}\right\}$ is regular. Then the following recurrence holds

$$
\phi_{n}(z)=\left(A_{n} Z_{n}(z)+B_{n} \frac{Z_{n}(z)}{Z_{n-2}(z)}\right) \phi_{n-1}(z)+C_{n} \frac{Z_{n}(z)}{Z_{n-2}(z)} \phi_{n-2}(z), \quad n=2,3, \ldots
$$

with constants $A_{n}, B_{n}, C_{n}$ satisfying the conditions

$$
\begin{aligned}
E_{n}=A_{n}+B_{n} / Z_{n-2}\left(\alpha_{n-1}\right) & \neq 0, & k=2,3, \ldots \\
C_{n} & \neq 0, & k=2,3, \ldots
\end{aligned}
$$

Let us introduce the real numbers

$$
D_{n}=\frac{1}{Z_{n-2}(z)}-\frac{1}{Z_{n-1}(z)}=-i \frac{\alpha_{n-1}-\alpha_{n-2}}{\left(1-\alpha_{n-1}\right)\left(1-\alpha_{n-2}\right)} \in \mathbb{R} .
$$

Since this is a constant not depending on $z$ and because $1 / Z_{k}\left(\alpha_{k}\right)=0$ for all $k$, we can use

$$
D_{n}=\frac{1}{Z_{n-2}\left(\alpha_{n-1}\right)}=-\frac{1}{Z_{n-1}\left(\alpha_{n-2}\right)} \in \mathbb{R}
$$

for any $n \geq 2$ when it will be convenient.

Lemma 2.3 Let $\kappa_{n}$ and $\kappa_{n}^{\prime}$ be as in (2.1). Define for $n \geq 1$

$$
E_{n}=\frac{1}{\kappa_{n-1}}\left[\kappa_{n}+\frac{\kappa_{n}^{\prime}}{Z_{n}\left(\alpha_{n-1}\right)}\right] \text {. }
$$

Then $E_{n} \in \mathbb{R}$.

If the recurrence relation holds, and hence $A_{n}$ and $B_{n}$ are defined, then the $E_{n}$ of (2.7) coincide with the $E_{n}$ of (2.4), i.e., $E_{n}=A_{n}+B_{n} D_{n}$. The latter also holds for $n=1$ if we set by definition $A_{1}=\kappa_{1}$ and $B_{1}=\kappa_{1}^{\prime}$.

Proof. Because the coefficients of the orthonormal functions are real and $D_{n}$ is real, it follows that also $E_{n}$ is real. To show that $E_{n}=A_{n}+B_{n} D_{n}$, we divide the recurrence relation (2.3) by $b_{n}(z)$ and set $z=\alpha_{n-1}$. With $(2.2)$ and $1 / Z_{k}\left(\alpha_{k}\right)=0$, we get the result.

Note that it easily follows from this definition of $E_{n}$ that $E_{n}=0$ if and only if $n$ is a singular index.

Lemma 2.4 Suppose the recurrence relation for the orthonormal functions $\phi_{n}$ holds with coefficients $A_{n}, B_{n}$ and $C_{n}$. Let $D_{n}$ be as in (2.6) and $E_{n}=A_{n}+B_{n} D_{n}$. Then

$$
E_{n}=-C_{n} E_{n-1}, \quad n \geq 2 .
$$

Proof. Because $b_{n-1} / Z_{n} \in \mathcal{L}_{n-1}$, it is orthogonal to $\phi_{n}$. Using the recurrence relation for $\phi_{n}$, we get

$$
\begin{aligned}
0 & =\left\langle\phi_{n}, b_{n-1} / Z_{n}\right\rangle \\
& =A_{n}\left\langle b_{n-1}, \phi_{n-1}\right\rangle+B_{n}\left\langle\frac{b_{n-1}}{Z_{n-2}}, \phi_{n-1}\right\rangle+C_{n}\left\langle\frac{b_{n-1}}{Z_{n-2}}, \phi_{n-2}\right\rangle .
\end{aligned}
$$


Because $\left\langle b_{n-2}, \phi_{n-1}\right\rangle=0$ and $\left\langle b_{i}, \phi_{i}\right\rangle=1 / \kappa_{i}$, we have

$$
0=\frac{A_{n}}{\kappa_{n-1}}+\frac{B_{n} D_{n}}{\kappa_{n-1}}+\frac{C_{n}}{\kappa_{n-2}}+C_{n} D_{n}\left\langle b_{n-1}, \phi_{n-2}\right\rangle .
$$

The remaining inner product can be evaluated when we use

$$
b_{n-1}(z)=\frac{\phi_{n-1}(z)}{\kappa_{n-1}}-\frac{\kappa_{n-1}^{\prime}}{\kappa_{n-1}} b_{n-2}(z)+\cdots
$$

so that

$$
\left\langle b_{n-1}, \phi_{n-2}\right\rangle=\left\langle\frac{\phi_{n-1}}{\kappa_{n-1}}, \phi_{n-2}\right\rangle-\frac{\kappa_{n-1}^{\prime}}{\kappa_{n-1}}\left\langle b_{n-2}, \phi_{n-2}\right\rangle=-\frac{\kappa_{n-1}^{\prime}}{\kappa_{n-1} \kappa_{n-2}} .
$$

When we combine (2.9) and (2.10), we get

$$
\begin{aligned}
0 & =\frac{A_{n}}{\kappa_{n-1}}+\frac{B_{n} D_{n}}{\kappa_{n-1}}+\frac{C_{n}}{\kappa_{n-2}}-\frac{C_{n} D_{n} \kappa_{n-1}^{\prime}}{\kappa_{n-1} \kappa_{n-2}} \\
& =\frac{A_{n}+B_{n} D_{n}}{\kappa_{n-1}}+\frac{C_{n}}{\kappa_{n-2}}\left[1-D_{n} \frac{\kappa_{n-1}^{\prime}}{\kappa_{n-1}}\right] \\
& =\frac{E_{n}}{\kappa_{n-1}}+\frac{C_{n}}{\kappa_{n-1}} \cdot \frac{1}{\kappa_{n-2}}\left[\kappa_{n-1}+\frac{\kappa_{n-1}^{\prime}}{Z_{n-1}\left(\alpha_{n-2}\right)}\right] \\
& =\frac{1}{\kappa_{n-1}}\left[E_{n}+C_{n} E_{n-1}\right],
\end{aligned}
$$

which gives us the expression we wanted.

For solutions of the recurrence relation, we can prove a general summation theorem. To formulate this formula, we define for $n \geq 1$

$$
H(z, w)=\frac{1}{Z_{n-1}(z)}-\frac{1}{Z_{n-1}(w)}=-i \frac{z-w}{(w-1)(z-1)} .
$$

Note that this expression does not depend on $n$. Furthermore we set

$$
\begin{aligned}
H_{n}(z, w)= & \frac{1}{Z_{n-2}(w) Z_{n-1}(z)}-\frac{1}{Z_{n-2}(z) Z_{n-1}(w)} \\
= & {\left[\frac{1}{Z_{n-2}(w) Z_{n-1}(z)}-\frac{1}{Z_{n-2}(w) Z_{n-2}(z)}\right] } \\
& +\left[\frac{1}{Z_{n-2}(w) Z_{n-2}(z)}-\frac{1}{Z_{n-2}(z) Z_{n-1}(w)}\right] \\
= & \frac{-D_{n}}{Z_{n-2}(w)}+\frac{D_{n}}{Z_{n-2}(z)}=D_{n} H(z, w) .
\end{aligned}
$$

Theorem 2.5 Let $x_{n}(z)$ and $y_{n}(z)$ be two solutions of the recurrence relation (2.3) and define

$$
F_{n}(z, w)=\frac{x_{n}(w) y_{n-1}(z)}{Z_{n}(w) Z_{n-1}(z)}-\frac{y_{n}(z) x_{n-1}(w)}{Z_{n}(z) Z_{n-1}(w)}
$$

Then, with $H(z, w)$ as in (2.11) and $E_{n}$ as in (2.7)

$$
\begin{aligned}
F_{n}(z, w) & =y_{n-1}(z) x_{n-1}(w) H(z, w) E_{n}-C_{n} F_{n-1}(z, w) \\
& =\left[\sum_{k=1}^{n-1} y_{k}(z) x_{k}(w)\right] H(z, w) E_{n}+(-1)^{n} C_{n} C_{n-1} \cdots C_{2} F_{1}(z, w) .
\end{aligned}
$$


Proof. We use the recurrence relation for $x_{n}$ and $y_{n}$ in the definition of $F_{n}(z, w)$, which gives

$$
\begin{aligned}
F_{n}(z, w)= & A_{n} x_{n-1}(w) y_{n-1}(z)\left[\frac{1}{Z_{n-1}(z)}-\frac{1}{Z_{n-1}(w)}\right] \\
& +B_{n} x_{n-1}(w) y_{n-1}(z)\left[\frac{1}{Z_{n-1}(z) Z_{n-2}(w)}-\frac{1}{Z_{n-1}(w) Z_{n-2}(z)}\right] \\
& -C_{n} F_{n-1}(z, w) .
\end{aligned}
$$

Using the expressions (2.11) and (2.12), we find

$$
\begin{aligned}
F_{n}(z, w) & =x_{n-1}(w) y_{n-1}(z) H(z, w)\left[A_{n}+B_{n} D_{n}\right]-C_{n} F_{n-1}(z, w) \\
& =x_{n-1}(w) y_{n-1}(z) H(z, w) E_{n}-C_{n} F_{n-1}(z, w)
\end{aligned}
$$

An induction argument leads to the result.

It is possible to derive from this formula the Christoffel-Darboux type formulas which are given below. However, this would require that the system $\phi_{n}$ is regular, since it is based on the existence of the recurrence relation. It is possible however to prove the ChristoffelDarboux formulas without using the recurrence relation and only relying on the orthogonality properties of the $\phi_{n}$. This is what we shall do here.

\section{Functions of the second kind}

With the orthonormal functions $\phi_{n}$, we associate functions of the second kind $\psi_{n}$ defined by

$$
\begin{aligned}
& \psi_{0}=-\frac{1-z}{1+z}=-\frac{Z_{0}(z)}{Z_{0}(0)} \\
& \psi_{n}=M_{t}\left\{D(t, z)\left[\phi_{n}(t)-\phi_{n}(z)\right]\right\}, \quad n \geq 1,
\end{aligned}
$$

where $D(t, z)$ is the Riesz-Herglotz kernel

$$
D(t, z)=\frac{t+z}{t-z} .
$$

We also introduced the notation $M_{t}$ to indicate that $M$ operates on its argument as a function of $t$. We prove that these functions of the second kind are also solutions of the recurrence (2.3).

Theorem 3.1 Suppose that the system of orthogonal rational functions $\phi_{n}$ is regular and let $\psi_{n}$ be the associated functions of the second kind. Then these $\psi_{n}$ satisfy the same recurrence relation (2.3) as the $\phi_{n}$.

Proof. We use the recurrence relation for $\phi_{n}(t)$ and $\phi_{n}(z)$ in the definition of $\psi_{n}$. This gives for $n \geq 2$

$$
\begin{aligned}
\psi_{n}(z)= & A_{n} M_{t}\left\{D(t, z)\left[Z_{n}(t) \phi_{n-1}(t)-Z_{n}(z) \phi_{n-1}(z)\right]\right\} \\
& +B_{n} M_{t}\left\{D(t, z)\left[\frac{Z_{n}(t)}{Z_{n-2}(t)} \phi_{n-1}(t)-\frac{Z_{n}(z)}{Z_{n-2}(z)} \phi_{n-1}(z)\right]\right\}
\end{aligned}
$$




$$
\begin{aligned}
& +C_{n} M_{t}\left\{D(t, z)\left[\frac{Z_{n}(t)}{Z_{n-2}(t)} \phi_{n-2}(t)-\frac{Z_{n}(z)}{Z_{n-2}(z)} \phi_{n-2}(z)\right]\right\} \\
= & A_{n} Z_{n}(z) \psi_{n-1}(z)+B_{n} \frac{Z_{n}(z)}{Z_{n-2}(z)} \psi_{n-1}(z)+C_{n} \frac{Z_{n}(z)}{Z_{n-2}(z)} \psi_{n-2}(z) \\
& +M_{t}\left\{D(t, z) f_{n}(t, z)\right\}+\delta_{n 2} \frac{Z_{2}(z)}{Z_{0}(0)} C_{2}
\end{aligned}
$$

with

$$
f_{n}(t, z)=A_{n}\left[Z_{n}(t)-Z_{n}(z)\right] \phi_{n-1}(t)+\left[\frac{Z_{n}(t)}{Z_{n-2}(t)}-\frac{Z_{n}(z)}{Z_{n-2}(z)}\right]\left[B_{n} \phi_{n-1}(t)+C_{n} \phi_{n-2}(t)\right]
$$

We note that

$$
Z_{n}(t)-Z_{n}(z)=-i \frac{(t-z)\left(\alpha_{n}-1\right)^{2}}{\left(t-\alpha_{n}\right)\left(z-\alpha_{n}\right)}
$$

and

$$
\frac{Z_{n}(t)}{Z_{n-2}(t)}-\frac{Z_{n}(z)}{Z_{n-2}(z)}=\frac{(t-z)\left(1-\alpha_{n}\right)\left(\alpha_{n-2}-\alpha_{n}\right)}{\left(t-\alpha_{n}\right)\left(z-\alpha_{n}\right)\left(1-\alpha_{n-2}\right)}
$$

Therefore

$$
f_{n}(t, z)=\frac{(t-z)\left(1-\alpha_{n}\right)}{\left(t-\alpha_{n}\right)\left(z-\alpha_{n}\right)}\left[A_{n} i\left(\alpha_{n}-1\right) \phi_{n-1}(t)+\frac{\alpha_{n-2}-\alpha_{n}}{1-\alpha_{n-2}}\left[B_{n} \phi_{n-1}(t)+C_{n} \phi_{n-2}(t)\right]\right]
$$

Next we split $D(t, z)$ as $D_{1}(t, z)+D_{2}(t, z)$ :

$$
D(t, z)=\frac{t-\alpha_{n}}{t-z}+\frac{z+\alpha_{n}}{t-z}
$$

Thus in the argument of $M_{t}$ in (3.1), the factor $t-z$ in the numerator of $f_{n}(t, z)$ cancels the denominator of $D(t, z)$.

Using the orthogonality of the $\phi_{k}$, we find

$$
\begin{aligned}
M_{t}\left\{D_{1}(t, z) f_{n}(t, z)\right\} & =0, \quad \text { for } n \geq 3 \\
& =C_{2} \frac{\left(\alpha_{2}-1\right)\left(1+\alpha_{2}\right)}{2\left(z-\alpha_{2}\right)}, \quad \text { for } n=2
\end{aligned}
$$

For the second term with $D_{2}(t, z)$, we use again the recurrence relation to write $f_{n}(t, z)$ as

$$
f_{n}(t, z)=\frac{t-z}{z-\alpha_{n}}\left[-\phi_{n}(t)-i\left(1-\alpha_{n}\right) A_{n} \phi_{n-1}(t)+\frac{1-\alpha_{n}}{1-\alpha_{n-2}}\left(B_{n} \phi_{n-1}(t)+C_{n} \phi_{n-2}(t)\right)\right] .
$$

Again, by the orthogonality of the $\phi_{k}$, we get

$$
\begin{aligned}
M_{t}\left\{D_{2}(t, z) f_{n}(t, z)\right\} & =0, \quad \text { for } n \geq 3 \\
& =C_{2} \frac{\left(z+\alpha_{2}\right)\left(1-\alpha_{2}\right)}{2\left(z-\alpha_{2}\right)}, \quad \text { for } n=2
\end{aligned}
$$

This proves the recurrence relation for $n \geq 3$ directly. For $n=2$, we can put together all the terms involved and we find that then also the recurrence relation is satisfied because

$$
\frac{Z_{2}(z)}{Z_{0}(0)} C_{2}-C_{2} \frac{\left(1-\alpha_{2}\right)\left(1+\alpha_{2}\right)}{2\left(z-\alpha_{2}\right)}+C_{2} \frac{\left(z+\alpha_{2}\right)\left(1-\alpha_{2}\right)}{2\left(z-\alpha_{2}\right)}=0 \text {. }
$$


It is possible to build some redundancy in the definition of the $\psi_{n}$. For an analogous proof in the case where the points $\alpha_{k}$ are inside the unit disk, see [7].

Lemma 3.2 Let $\phi_{n}$ be the orthonormal system and $\psi_{n}$ the associated functions of the second kind. For $n>0$ and for any $f$ such that (as a function of $t$ ) $D(t, z)[f(t)-f(z)] \in \mathcal{L}_{n-1}$ we have

$$
\psi_{n}(z) f(z)=M_{t}\left\{D(t, z)\left[\phi_{n}(t) f(t)-\phi_{n}(z) f(z)\right]\right\}
$$

Proof. Since

$$
M_{t}\left\{D(t, z)\left[f(t) \phi_{n}(t)-f(z) \phi_{n}(z)\right]\right\}=f(z) \psi_{n}(z)+M_{t}\left\{D(t, z)[f(t)-f(z)] \phi_{n}(t)\right\},
$$

the result follows by the orthogonality of the $\phi_{n}$.

Note that in particular we could take $f \in \mathcal{L}_{n-1}$ or $f(t)=g(t)\left(t-\alpha_{n}\right) /(t+z)$ with $g \in \mathcal{L}_{n}$,

We shall now derive a Liouville-Ostrogradskii type determinant formula.

Theorem 3.3 (determinant formula) Let $\phi_{n}$ be the orthonormal functions and $\psi_{n}$ the functions of the second kind, then for $n \geq 1$ we have with $E_{n}$ as in (2.7)

$$
\begin{aligned}
\phi_{n-1}(z) \psi_{n}(z)-\phi_{n}(z) \psi_{n-1}(z) & =\frac{2 i z}{(1-z)^{2}} E_{n} Z_{n-1}(z) Z_{n}(z) \\
& =-\frac{2 i z\left(\alpha_{n}-1\right)\left(\alpha_{n-1}-1\right) E_{n}}{\left(z-\alpha_{n-1}\right)\left(z-\alpha_{n}\right)}
\end{aligned}
$$

Proof. We note that

$$
\begin{aligned}
& \phi_{n-1}(z)\left[\phi_{n}(t)-\phi_{n}(z)\right]-\phi_{n}(z)\left[\phi_{n-1}(t)-\phi_{n-1}(z)\right] \\
& \quad=\phi_{n-1}(t)\left[\phi_{n}(t)-\phi_{n}(z)\right]-\phi_{n}(t)\left[\phi_{n-1}(t)-\phi_{n-1}(z)\right] .
\end{aligned}
$$

Multiply with $D(t, z)$ and apply $M_{t}$ to get for the left-hand side

$$
\phi_{n-1}(z) \psi_{n}(z)-\phi_{n}(z) \psi_{n-1}(z)
$$

while for the right-hand side we find

$$
M_{t}\left\{\phi_{n-1}(t) D(t, z)\left[\phi_{n}(t)-\phi_{n}(z)\right]\right\}-M_{t}\left\{\phi_{n}(t) D(t, z)\left[\phi_{n-1}(t)-\phi_{n-1}(z)\right]\right\} .
$$

Note that in the second term $D(t, z)\left[\phi_{n-1}(t)-\phi_{n-1}(z)\right] \in \mathcal{L}_{n-1}$ so that this term is zero by the orthogonality of $\phi_{n}$. To compute the first term, we define

$$
h(t)=D(t, z)\left[\phi_{n}(t)-\phi_{n}(z)\right]=\gamma_{n} b_{n}(t)+\gamma_{n}^{\prime} b_{n-1}(t)+\cdots
$$

where

$$
\gamma_{n}=\left[\frac{h(t)}{b_{n}(t)}\right]_{t=\alpha_{n}}=D\left(\alpha_{n}, z\right) \kappa_{n}
$$

and

$$
\gamma_{n}+\frac{\gamma_{n}^{\prime}}{Z_{n}\left(\alpha_{n-1}\right)}=\left[\frac{h(t)}{b_{n}(t)}\right]_{t=\alpha_{n-1}}=D\left(\alpha_{n-1}, z\right)\left[\kappa_{n}+\frac{\kappa_{n}^{\prime}}{Z_{n}\left(\alpha_{n-1}\right)}\right]=D\left(\alpha_{n-1}, z\right) \kappa_{n-1} E_{n}
$$


Thus, by the orthogonality of $\phi_{n-1}=\phi_{(n-1) *}$

$$
M\left\{\phi_{n-1} h\right\}=\gamma_{n} M\left\{\phi_{n-1} b_{n}\right\}+\gamma_{n}^{\prime} M\left\{\phi_{n-1} b_{n-1}\right\} .
$$

Because $\phi_{k}=\kappa_{k} b_{k}+\kappa_{k}^{\prime} b_{k-1}+\cdots$, it follows by orthogonality that

$$
M\left\{\phi_{n-1} b_{n}\right\}=-\frac{\kappa_{n}^{\prime}}{\kappa_{n} \kappa_{n-1}} \quad \text { and } \quad M\left\{\phi_{n-1} b_{n-1}\right\}=\frac{1}{\kappa_{n-1}} .
$$

So we obtain

$$
\begin{aligned}
M\left\{\phi_{n-1} h\right\} & =-\gamma_{n} \frac{\kappa_{n}^{\prime}}{\kappa_{n} \kappa_{n-1}}+\frac{\gamma_{n}^{\prime}}{\kappa_{n-1}} \\
& =-\frac{\gamma_{n} \kappa_{n}^{\prime}}{\kappa_{n} \kappa_{n-1}}+\left[D\left(\alpha_{n-1}, z\right) E_{n}-\frac{\gamma_{n}}{\kappa_{n-1}}\right] Z_{n}\left(\alpha_{n-1}\right) \\
& =-D\left(\alpha_{n}, z\right) \frac{\kappa_{n}^{\prime}}{\kappa_{n-1}}+D\left(\alpha_{n-1}, z\right) E_{n} Z_{n}\left(\alpha_{n-1}\right)-D\left(\alpha_{n}, z\right) \kappa_{n} Z_{n}\left(\alpha_{n-1}\right) \\
& =-D\left(\alpha_{n}, z\right) E_{n} Z_{n}\left(\alpha_{n-1}\right)+D\left(\alpha_{n-1}, z\right) E_{n} Z_{n}\left(\alpha_{n-1}\right) \\
& =E_{n} Z_{n}\left(\alpha_{n-1}\right)\left[D\left(\alpha_{n-1}, z\right)-D\left(\alpha_{n}, z\right)\right] .
\end{aligned}
$$

Working this out gives the result.

\section{Christoffel-Darboux relations}

We now prove some Chistoffel-Darboux type relations.

Theorem 4.1 (Christoffel-Darboux relation) Suppose $\phi_{n}$ are the orthonormal functions and let $H(z, w)$ and $E_{n}$ be defined by (2.11) and (2.7). Then

$$
\frac{\phi_{n}(w) \phi_{n-1}(z)}{Z_{n}(w) Z_{n-1}(z)}-\frac{\phi_{n}(z) \phi_{n-1}(w)}{Z_{n}(z) Z_{n-1}(w)}=H(z, w) E_{n} \sum_{k=0}^{n-1} \phi_{k}(z) \phi_{k}(w) .
$$

Proof. Define

$$
g(z, w)=\left(w-\alpha_{n}\right)\left(z-\alpha_{n-1}\right) \phi_{n}(w) \phi_{n-1}(z) \quad \text { and } \quad G(w)=g(z, w)-g(w, z) .
$$

Then the Christoffel-Darboux relation which has to be shown is equivalent with

$$
F(w)=\frac{G(w)}{z-w}=-i\left(\alpha_{n}-1\right)\left(\alpha_{n-1}-1\right) E_{n} \sum_{k=0}^{n-1} \phi_{k}(z) \phi_{k}(w)
$$

Observe that $F(w) \in \mathcal{L}_{n-1}$ so that it can be written as

$$
F(w)=\sum_{k=0}^{n-1} \gamma_{k}(z) \phi_{k}(w), \quad \gamma_{k}(z)=M\left\{F \phi_{k *}\right\}=M\left\{F \phi_{k}\right\}
$$

We have

$$
\gamma_{k}(z)=M\left\{F \phi_{k}\right\}=M_{w}\left\{F(w)\left[\phi_{k}(w)-\phi_{k}(z)\right]\right\}+\phi_{k}(z) M\{F\}
$$


The first term is zero. To see this, we write it out as

$$
\begin{aligned}
M_{w}\left\{F(w)\left[\phi_{k}(w)-\phi_{k}(z)\right]\right\}= & \left(z-\alpha_{n-1}\right) \phi_{n-1}(z) M_{w}\left\{\phi_{n}(w) \frac{w-\alpha_{n}}{z-w}\left[\phi_{k}(w)-\phi_{k}(z)\right]\right\} \\
& -\left(z-\alpha_{n}\right) \phi_{n}(z) M_{w}\left\{\phi_{n-1}(w) \frac{w-\alpha_{n-1}}{z-w}\left[\phi_{k}(w)-\phi_{k}(z)\right]\right\} .
\end{aligned}
$$

Because $\phi_{n} \perp \mathcal{L}_{n-1}$, the first term is zero and because $\phi_{n-1} \perp \mathcal{L}_{n-2}$, the second term is zero. Thus it remains that $\gamma_{k}(z)=\phi_{k}(z) M\{F\}$. We note that

$$
M\{F\}=\left(z-\alpha_{n-1}\right) \phi_{n-1}(z) f_{n}(z)-\left(z-\alpha_{n}\right) \phi_{n}(z) f_{n-1}(z), \quad f_{i}(z)=M_{w}\left\{\frac{w-\alpha_{i}}{z-w} \phi_{i}(w)\right\} .
$$

By adding and subtracting $\left(z-\alpha_{n-1}\right)\left(z-\alpha_{n}\right) \phi_{n-1}(z) \phi_{n}(z) D(z, w) /(2 z)$, we can rewrite this as

$$
M\{F\}=\left(z-\alpha_{n-1}\right) \phi_{n-1}(z) g_{n}(z)-\left(z-\alpha_{n}\right) \phi_{n}(z) g_{n-1}(z)
$$

with for $i=n, n-1>0$

$$
g_{i}(z)=M_{w}\left\{D(z, w)\left[\frac{w-\alpha_{i}}{z+w} \phi_{i}(w)-\frac{z-\alpha_{i}}{2 z} \phi_{i}(z)\right]\right\}=\frac{z-\alpha_{i}}{2 z} \psi_{i}(z)
$$

(the last equality is by Lemma 3.2). For $i=0$, one has to use $\phi_{0}=1, \alpha_{0}=-1$ and $\psi_{0}(z)=(z-1) /(z+1)$ and it then follows that the previous relation also holds for $i=0$. Thus, using the determinant relation of Theorem 3.3, this gives

$$
\begin{aligned}
M\{F\} & =\frac{\left(z-\alpha_{n}\right)\left(z-\alpha_{n-1}\right)}{2 z}\left[\phi_{n-1}(z) \psi_{n}(z)-\phi_{n}(z) \psi_{n-1}(z)\right] \\
& =-i\left(\alpha_{n}-1\right)\left(\alpha_{n-1}-1\right) E_{n}
\end{aligned}
$$

so that eventually

$$
F(w)=\sum_{k=0}^{n-1} \gamma_{k}(z) \phi_{k}(w)=M\{F\} \sum_{k=0}^{n-1} \phi_{k}(z) \phi_{k}(w)
$$

yields (4.2).

We also have the following generalization of the determinant formula.

Theorem 4.2 Let $\left\{\phi_{n}\right\}$ be the orthonormal functions and $\psi_{n}$ the associated functions of the second kind. Define

$$
F_{n}(z, w)=\frac{\psi_{n}(w) \phi_{n-1}(z)}{Z_{n}(w) Z_{n-1}(z)}-\frac{\phi_{n}(z) \psi_{n-1}(w)}{Z_{n}(z) Z_{n-1}(w)}
$$

Let $H(z, w)$ be as in (2.11), $E_{n}$ as in (2.7) and $D(z, w)$ the Riesz-Herglotz kernel. Then, for $w \neq z$,

$$
F_{n}(z, w)=\left[\sum_{k=1}^{n-1} \phi_{k}(z) \psi_{k}(w)-D(z, w)\right] H(z, w) E_{n}
$$

For $w=z$, this reduces to the determinant formula. 
Proof. Define $g(z, w)$ as in (4.1) and set

$$
G(z, w)=g(z, w)-g(w, z) .
$$

Then, by the Chistoffel-Darboux formula

$$
G(z, w)=-i(z-w)\left(\alpha_{n}-1\right)\left(\alpha_{n-1}-1\right) E_{n} \sum_{k=0}^{n-1} \phi_{k}(z) \phi_{k}(w)
$$

Apply $M_{t}$ on the expression

$$
\frac{D(t, w)}{t+w} G(t, z)-\frac{D(t, w)}{2 w} G(w, z)
$$

and this gives with (4.3)

$$
\begin{aligned}
& \left(z-\alpha_{n}\right)\left(w-\alpha_{n-1}\right) \phi_{n}(z) \psi_{n-1}(w)-\left(w-\alpha_{n}\right)\left(z-\alpha_{n-1}\right) \psi_{n}(w) \phi_{n-1}(z) \\
& =-i\left(\alpha_{n}-1\right)\left(\alpha_{n-1}-1\right) E_{n}\left[\sum_{k=1}^{n-1} \phi_{k}(z)(w-z) \psi_{k}(w)+(z+w)\right] \\
& =i\left(\alpha_{n}-1\right)\left(\alpha_{n-1}-1\right)(z-w) E_{n}\left[\sum_{k=1}^{n-1} \phi_{k}(z) \psi_{k}(w)-D(z, w)\right]
\end{aligned}
$$

This can be rewritten in the required form.

An analog of the Christoffel-Darboux relations for the functions of the second kind may also be obtained.

Theorem 4.3 Let $\psi_{n}$ be the functions of the second kind. Then, in analogy with the Christoffel-Darboux relation we have

$$
\frac{\psi_{n}(w) \psi_{n-1}(z)}{Z_{n}(w) Z_{n-1}(z)}-\frac{\psi_{n}(z) \psi_{n-1}(w)}{Z_{n}(z) Z_{n-1}(w)}=H(z, w) E_{n}\left[\sum_{k=1}^{n-1} \psi_{k}(z) \psi_{k}(w)-1\right]
$$

with $H(z, w)$ as in (2.11) and $E_{n}$ as in (2.7).

Proof. We set

$$
G(z, w)=\left(w-\alpha_{n}\right)\left(z-\alpha_{n-1}\right) \phi_{n}(w) \psi_{n-1}(z)-\left(z-\alpha_{n}\right)\left(w-\alpha_{n-1}\right) \phi_{n-1}(w) \psi_{n}(z) .
$$

Then by the previous theorem

$$
G(z, w)=i\left(\alpha_{n}-1\right)\left(\alpha_{n-1}-1\right)(z-w) E_{n}\left[\sum_{k=1}^{n-1} \phi_{k}(w) \psi_{k}(z)-D(z, w)\right] .
$$

By applying $M_{t}$ on

$$
\frac{D(t, w)}{t+w} G(z, t)-\frac{D(t, w)}{2 w} G(z, w)
$$

we get by using (4.3)

$$
\begin{aligned}
& \left(w-\alpha_{n}\right)\left(z-\alpha_{n-1}\right) \psi_{n}(w) \psi_{n-1}(z)-\left(z-\alpha_{n}\right)\left(w-\alpha_{n-1}\right) \psi_{n-1}(w) \psi_{n}(z) \\
& =-i\left(\alpha_{n}-1\right)\left(\alpha_{n-1}-1\right)(z-w) E_{n}\left[\sum_{k=1}^{n-1} \psi_{k}(z) \psi_{k}(w)-1\right]
\end{aligned}
$$

and this is equivalent with the required formula.

Finally, we give a combination of the previous summation formulas. 
Theorem 4.4 Let $\phi_{n}$ be the orthonormal functions, $\psi_{n}$ the functions of the second kind and define for an arbitrary complex parameter $s$

$$
\chi_{n}(z ; s)=\psi_{n}(z)+s \phi_{n}(z) .
$$

Then

$$
\begin{aligned}
& \frac{\chi_{n}(w ; t) \chi_{n-1}(z ; s)}{Z_{n}(w) Z_{n-1}(z)}-\frac{\chi_{n}(z ; s) \chi_{n-1}(w ; t)}{Z_{n}(z) Z_{n-1}(w)} \\
& \quad=H(z, w) E_{n}\left[\sum_{k=1}^{n-1} \chi_{k}(z ; s) \chi_{k}(w ; t)+[s t-1+D(z, w)(t-s)]\right]
\end{aligned}
$$

with $H(z, w)$ as in (2.11) and $E_{n}$ as in (2.7).

Proof. This is directly obtained by working out the left-hand side and using the three previous theorems.

\section{Green's formula}

We now give complex forms of the previous Christoffel-Darboux type formulas. Therefore we introduce

$$
\tilde{H}(z, w)=\frac{1}{Z_{n}(z)}-\frac{1}{\overline{Z_{n}(w)}}=-\frac{i(1-z \bar{w})}{(1-z)(1-\bar{w})}, \quad n \geq 0
$$

Note that $\tilde{H}(z, w)=H_{*}(z, w)$ where the substar is with respect to $z$. For $w=z$, we have $\tilde{H}(z, z)=-i P(1, z)$ with

$$
P(t, z)=\frac{1-|z|^{2}}{|t-z|^{2}}
$$

the Poisson kernel. Furthermore we define for $n \geq 2$

$$
\tilde{H}_{n}(z, w)=\frac{1}{\overline{Z_{n-2}(w)} Z_{n-1}(z)}-\frac{1}{\overline{Z_{n-1}(w)} Z_{n-2}(z)}=D_{n} \tilde{H}(z, w) .
$$

We now give without proof the following complex analog of Theorem 2.5. A possible proof would be to take substar conjugates of the relations from the previous section. Note that we then need the fact that the numbers $E_{n}$ and $C_{n}$ are real, which they are by Lemma 2.3 and Lemma 2.4 .

Theorem 5.1 (Green's formula) Let $x_{n}(z)$ and $y_{n}(z)$ be both solutions of the recurrence relation (2.3) and define

$$
G_{n}(z, w)=\overline{\left(\frac{x_{n}(w)}{Z_{n}(w)}\right)}\left(\frac{y_{n-1}(z)}{Z_{n-1}(z)}\right)-\left(\frac{y_{n}(z)}{Z_{n}(z)}\right) \overline{\left(\frac{x_{n-1}(w)}{Z_{n-1}(w)}\right)} .
$$

Then, with $\tilde{H}(z, w)$ as in (5.1) and $E_{n}$ as in (2.7)

$$
\begin{aligned}
G_{n}(z, w) & =y_{n-1}(z) \overline{x_{n-1}(w)} \tilde{H}(z, w) E_{n}-C_{n} G_{n-1}(z, w) \\
& =\left[\sum_{k=1}^{n-1} y_{k}(z) \overline{x_{k}(w)}\right] \tilde{H}(z, w) E_{n}+(-1)^{n} C_{n} C_{n-1} \cdots C_{2} G_{1}(z, w) .
\end{aligned}
$$


One can use this theorem and take $x_{k}=y_{k}=\phi_{k}$ or $x_{k}=y_{k}=\psi_{k}$ or $x_{k}=\phi_{k}$ and $y_{k}=\psi_{k}$. One then obtains the formulas in the theorem below which are then proved under the assumpsion that they satisfy the recurrence relation, in particular that the $\phi_{k}$ form a regular system. However, it is equally simple to follow the way of reasoning used in the previous section to arrive at the Christoffel-Darboux type formulas where orthogonality was the only assumption needed, and again one will arrive at the results of the theorem below. Thus the results hold without assumimg that there is a recurrence relation, i.e., without the system $\phi_{n}$ being regular. We just give the result without proof.

Theorem 5.2 Let $\phi_{n}$ be the orthonormal functions and $\psi_{n}$ the functions of the second kind. Let $\tilde{H}(z, w)$ be as defined in (5.1), $E_{n}$ as in (2.7) and $D(z, w)$ the Riesz-Herglotz kernel. Then

$$
\begin{aligned}
& \overline{\left(\frac{\phi_{n}(w)}{Z_{n}(w)}\right)}\left(\frac{\phi_{n-1}(z)}{Z_{n-1}(z)}\right)-\left(\frac{\phi_{n}(z)}{Z_{n}(z)} \overline{\left(\frac{\phi_{n-1}(w)}{Z_{n-1}(w)}\right)}=\left[\sum_{k=0}^{n-1} \phi_{k}(z) \overline{\phi_{k}(w)}\right] \tilde{H}(z, w) E_{n}\right. \\
& \overline{\left(\frac{\psi_{n}(w)}{Z_{n}(w)}\right)}\left(\frac{\psi_{n-1}(z)}{Z_{n-1}(z)}\right)-\left(\frac{\psi_{n}(z)}{Z_{n}(z)}\right) \overline{\left(\frac{\psi_{n-1}(w)}{Z_{n-1}(w)}\right)}=\left[\sum_{k=1}^{n-1} \psi_{k}(z) \overline{\psi_{k}(w)}+1\right] \tilde{H}(z, w) E_{n} \\
& \overline{\left(\frac{\psi_{n}(w)}{Z_{n}(w)}\right)}\left(\frac{\phi_{n-1}(z)}{Z_{n-1}(z)}\right)-\left(\frac{\phi_{n}(z)}{Z_{n}(z)}\right) \overline{\left(\frac{\psi_{n-1}(w)}{Z_{n-1}(w)}\right)}=\left[\sum_{k=1}^{n-1} \phi_{k}(z) \overline{\psi_{k}(w)}-D_{*}(z, w)\right] \tilde{H}(z, w) E_{n},
\end{aligned}
$$

where in the last equation, the substar is with respect to $z$.

Note that this also holds for $w=z$. In that case

$$
D_{*}(z, z)=\frac{1+|z|^{2}}{1-|z|^{2}}
$$

The previous relations can be combined to give the following

Theorem 5.3 Let $\phi_{n}$ be the orthonormal functions and $\psi_{n}$ the functions of the second kind. For an arbitrary complex $s$, we set

$$
\chi_{n}(z ; s)=\psi_{n}(z)+s \phi_{n}(z)
$$

Then

$$
\begin{aligned}
& \overline{\left(\frac{\chi_{n}(w ; t)}{Z_{n}(w)}\right)}\left(\frac{\chi_{n-1}(z ; s)}{Z_{n-1}(z)}\right)-\left(\frac{\chi_{n}(z ; s)}{Z_{n}(z)}\right) \overline{\left(\frac{\chi_{n-1}(w ; t)}{Z_{n-1}(w)}\right)} \\
& =\left[\sum_{k=1}^{n-1} \chi_{k}(z ; s) \overline{\chi_{k}(w, t)}+1+s \bar{t}-(s+\bar{t}) D_{*}(z, w)\right] \tilde{H}(z, w) E_{n}
\end{aligned}
$$

In particular for $z=w$ and $t=s$, we obtain

$$
\begin{aligned}
& \overline{\left(\frac{\chi_{n}(z ; s)}{Z_{n}(z)}\right)}\left(\frac{\chi_{n-1}(z ; s)}{Z_{n-1}(z)}\right)-\left(\frac{\chi_{n}(z ; s)}{Z_{n}(z)}\right) \overline{\left(\frac{\chi_{n-1}(z ; s)}{Z_{n-1}(z)}\right)} \\
& =\left[\sum_{k=1}^{n-1}\left|\chi_{k}(z ; s)\right|^{2}+|1-s|^{2}\right] \tilde{H}(z, z) E_{n}+(s+\bar{s}) E_{n} \frac{2 i|z|^{2}}{|z-1|^{2}} .
\end{aligned}
$$


Proof. By the previous theorem, we can evaluate the left-hand side and obtain the formula (5.3). For $z=w$ and $t=s$, this equals

$$
\left[\sum_{k=1}^{n-1}\left|\chi_{k}(z ; s)\right|^{2}\right] \tilde{H}(z, z) E_{n}+E_{n} Y(z, s)
$$

with

$$
\begin{aligned}
Y(z, s) & =\tilde{H}(z, z)\left[1-(s+\bar{s}) D_{*}(z, z)+|s|^{2}\right] \\
& =\tilde{H}(z, z)|1-s|^{2}+(s+\bar{s}) \tilde{H}(z, z)\left[1-D_{*}(z, z)\right] .
\end{aligned}
$$

Because

$$
\tilde{H}(z, z)\left[1-D_{*}(z, z)\right]=i \frac{1-|z|^{2}}{|1-z|^{2}}\left[\frac{1+|z|^{2}}{1-|z|^{2}}-1\right]=i \frac{2|z|^{2}}{|1-z|^{2}} .
$$

we get the result (5.4).

\section{Quasi-orthogonal functions}

We define quasi-orthogonal functions as

$$
Q_{n}(z, \tau)=\phi_{n}(z)+\tau \frac{Z_{n}(z)}{Z_{n-1}(z)} \phi_{n-1}(z), \quad \tau \in \hat{\mathbb{R}}=\mathbb{R} \cup\{\infty\}, n \geq 1 .
$$

For $\tau=\infty$, we set

$$
Q_{n}(z, \infty)=\frac{Z_{n}(z)}{Z_{n-1}(z)} \phi_{n-1}(z)
$$

It was proved in [3] that if $\phi_{n}$ is regular, then there always exists infinitely many so called regular values $\tau=\tau_{n} \in \hat{\mathbb{R}}$ such that $Q_{n}\left(z, \tau_{n}\right)$ has $n$ simple zeros, all lying on $\mathbb{T} \backslash\left\{\alpha_{1}, \alpha_{2}, \ldots, \alpha_{n}\right\}$. In fact all, except finitely many $\tau \in \hat{\mathbb{R}}$ are regular values. Suppose we have chosen a regular value $\tau_{n}$ and suppose that the zeros of $Q_{n}\left(z, \tau_{n}\right)$ are given by $\xi_{k}=\xi_{n k}(\tau), k=1, \ldots, n$. They can be used to construct quadrature formulas. These formulas use the $\xi_{k}$ for their abscissas and their weights $\lambda_{n k}(\tau)$ are given by

$$
\lambda_{n k}(\tau)=M\left\{L_{n k}\right\}=M\left\{L_{n k}(z, \tau) L_{n k *}(z, \tau)\right\}>0
$$

with

$$
L_{n k}(z, \tau)=\frac{\left(z-\xi_{1}\right) \cdots\left(z-\xi_{k-1}\right)\left(z-\xi_{k+1}\right) \cdots\left(z-\xi_{n}\right) \omega_{n-1}\left(\xi_{k}\right)}{\left(\xi_{k}-\xi_{1}\right) \cdots\left(\xi_{k}-\xi_{k-1}\right)\left(\xi_{k}-\xi_{k+1}\right) \cdots\left(\xi_{k}-\xi_{n}\right) \omega_{n-1}(z)} .
$$

It was also proved that these quadrature formulas are exact in $\mathcal{R}_{n-1}=\mathcal{L}_{n-1} \cdot \mathcal{L}_{n-1}$.

Theorem 6.1 With the notation just introduced, we have that

$$
M\{f\}=\sum_{k=1}^{n} \lambda_{n k}(\tau) f\left(\xi_{n k}(\tau)\right)
$$

for all $f \in \mathcal{R}_{n-1}=\mathcal{L}_{n-1} \cdot \mathcal{L}_{n-1}$. 
Alternative expressions for the weights can be obtained as follows. First introduce quasiorthogonal functions of the second kind by

$$
P_{n}(z)=P_{n}(z, \tau)=\psi_{n}(z)+\tau \frac{Z_{n}(z)}{Z_{n-1}(z)} \psi_{n-1}(z), \quad \tau \in \hat{\mathbb{R}}, n \geq 1
$$

We then have

Theorem 6.2 The weights of the quadrature formula are given by

$$
\lambda_{n k}=\frac{1}{2 \xi_{k}} \frac{P_{n}\left(\xi_{k}\right)}{Q_{n}^{\prime}\left(\xi_{k}\right)}
$$

where the prime means derivative.

Proof. From the partial fraction decomposition we see that

$$
R_{n}(z)=-\frac{P_{n}(z)}{Q_{n}(z)}=\sum_{j=1}^{n} \lambda_{n j} D\left(\xi_{j}, z\right)
$$

with $D(t, z)$ the Riesz-Herglotz kernel. Therefore

$$
\left(z-\xi_{k}\right) R_{n}(z)=\sum_{j \neq k} \lambda_{n j} D\left(\xi_{j}, z\right)\left(z-\xi_{k}\right)+\lambda_{n k} D\left(\xi_{k}, z\right)\left(z-\xi_{k}\right)
$$

Taking the limit for $z \rightarrow \xi_{k}$ cancels the sum and we get

$$
-\frac{P_{n}\left(\xi_{k}\right)}{Q_{n}^{\prime}\left(\xi_{k}\right)}=\lambda_{n k} \lim _{z \rightarrow \xi_{k}} D\left(\xi_{k}, z\right)\left(z-\xi_{k}\right)
$$

and this gives the result.

Another expression, which clearly shows the positivity of the weights is

Theorem 6.3 The weights of the quadrature formula are given by

$$
\lambda_{n k}=\left[\sum_{j=0}^{n-1}\left|\phi_{j}\left(\xi_{k}\right)\right|^{2}\right]^{-1} .
$$

Proof. Since $Q_{n}\left(\xi_{k}\right)=0$, we get from the first formula in Theorem 5.2 with $w=\xi_{k}$ and $z=t$

$$
\frac{\overline{\phi_{n-1}\left(\xi_{k}\right)}}{\overline{Z_{n-1}\left(\xi_{k}\right)} Z_{n}(t)}\left[Q_{n}\left(\xi_{k}\right)-Q_{n}(t)\right]=\left[\sum_{j=0}^{n-1} \phi_{j}(t) \overline{\phi_{j}\left(\xi_{k}\right)}\right] \tilde{H}\left(t, \xi_{k}\right) E_{n} .
$$

Dividing by $\xi_{k}-t$ and letting $t$ tend to $\xi_{k}$ we get

$$
\frac{\overline{\phi_{n-1}\left(\xi_{k}\right)}}{\overline{Z_{n-1}\left(\xi_{k}\right)} Z_{n}\left(\xi_{k}\right)} Q_{n}^{\prime}\left(\xi_{k}\right)=k_{n-1}\left(\xi_{k}, \xi_{k}\right) E_{n} \lim _{t \rightarrow \xi_{k}} \frac{\tilde{H}\left(t, \xi_{k}\right)}{\xi_{k}-t}
$$

where $k_{n-1}(z, z)=\sum_{j=0}^{n-1}\left|\phi_{j}(z)\right|^{2}$ and the limit in the right-hand side is $i /\left(1-\xi_{k}\right)^{2}$, provided that $\xi_{k} \neq 1$. 
Similarly, using the third formula in Theorem 5.2 with $z=w=\xi_{k}$ and taking into account that $Q_{n}\left(\xi_{k}\right)=0$, we get

$$
\frac{\overline{\phi_{n-1}\left(\xi_{k}\right)}}{\overline{Z_{n-1}\left(\xi_{k}\right)} Z_{n}\left(\xi_{k}\right)} P_{n}\left(\xi_{k}\right)=-E_{n} \lim _{t \rightarrow \xi_{k}} \overline{D_{*}\left(t, \xi_{k}\right)} \overline{\tilde{H}\left(t, \xi_{k}\right)}
$$

where the limit is (again for $\xi_{k} \neq 1$ )

$$
-i \frac{2}{\left|1-\xi_{k}\right|^{2}}
$$

We now plug the values of $Q_{n}^{\prime}\left(\xi_{k}\right)$ and $P_{n}\left(\xi_{k}\right)$ into the expression of $\lambda_{n k}$ of the previous theorem and we get the result for $\xi_{k} \neq 1$.

For $\xi_{k}=1$, we use a perturbation technique. First we choose a continuous perturbation $\tau(\epsilon) \in \mathbb{R}$ of the parameter $\tau$ in $Q_{n}$. Assume that $\lim _{\epsilon \rightarrow 0} \tau(\epsilon)=\tau$. The coefficients of $Q_{n}$ depend continuously on $\epsilon$ and hence also its zero $\xi_{k}$. Therefore we can always choose $\epsilon \neq 0$ such that $\xi_{k}(\epsilon) \neq 1$. Then the relation we want does holds true by our previous arguments. Since $\xi_{k}(\epsilon) \rightarrow 1$ as $\epsilon \rightarrow 0$ and since both $P_{n}$ and $Q_{n}^{\prime}$ as well as their ratio depend continuously on $\epsilon$ in the neighbourhood of $\epsilon=0$, we finally obtain the result for $\xi_{k}=1$ since

$$
\frac{1}{k_{n-1}(1,1)}=\lim _{\epsilon \rightarrow 0} \frac{1}{k_{n-1}\left(\xi_{k}(\epsilon), \xi_{k}(\epsilon)\right)}=\lim _{\epsilon \rightarrow 0} \frac{1}{2 \xi_{k}(\epsilon)} \frac{P_{n}\left(\xi_{k}(\epsilon)\right)}{Q_{n}^{\prime}\left(\xi_{k}(\epsilon)\right)}=\frac{1}{2} \frac{P_{n}(1)}{Q_{n}^{\prime}(1)}=\lambda_{n k}
$$

This concludes the proof.

The discrete measure which takes weights $\lambda_{n k}(\tau)$ in the points $\xi_{n k}(\tau), k=1, \ldots, n$ will be denoted by $\mu_{n}=\mu_{n}(\cdot, \tau)$. The set of regular $\tau$ values for which such a measure exists is dense in $\mathbb{R}$.

\section{$7 \quad$ Nested disks}

In this section we use the notation

$$
s=s_{n}(z)=R_{n}(z, \tau)=-\frac{P_{n}(z, \tau)}{Q_{n}(z, \tau)}
$$

where $Q_{n}$ are the quasi-orthogonal functions and $P_{n}$ are the quasi-orthogonal functions of the second kind. Obviously when $n$ is a regular index, then $\tau \rightarrow s_{n}(z)$ maps (for a fixed $z \in \hat{\mathbb{C}} \backslash \mathbb{T}$ with $\hat{\mathbb{C}}=\mathbb{C} \cup\{\infty\})$ the extended real line $\hat{\mathbb{R}}$ onto a circle $K_{n}(z)$. Furthermore, the upper half plane is mapped onto its interior. This follows for instance from Theorem 7.1(2) below. The closed disk with boundary $K_{n}(z)$ is denoted as $\Delta_{n}(z)$.

For $z \in \mathbb{T}$, the circle $K_{n}(z)$ degenerates to a line (the imaginary axis).

When $n$ is a singular index, then the transformation is degenerate. In that case, the whole plane is mapped to a point. Indeed, since for a singular $n$ we have $E_{n}=0$, it follows from the Christoffel-Darboux relation that

$$
\frac{\phi_{n}(w)}{Z_{n}(w)} \frac{\phi_{n-1}(z)}{Z_{n-1}(z)}=\frac{\phi_{n}(z)}{Z_{n}(z)} \frac{\phi_{n-1}(w)}{Z_{n-1}(w)}
$$


for any $z$ and $w$. Choose $w$ such that $\phi_{n-1}(w) / Z_{n-1}(w) \neq 0$, then we see that there should be a constant $c_{n}$ such that

$$
\phi_{n}(z)=c_{n} \frac{Z_{n}(z)}{Z_{n-1}(z)} \phi_{n-1}(z) .
$$

It follows similarly from Theorem 4.3 that the same holds for the functions of the second kind $\psi_{n}$, i.e., we may replace $\phi$ by $\psi$ in the previous relation and the constant $c_{n}$ is the same for $\phi$ and $\psi$ as follows from the determinant fromula of Theorem 3.3. Hence we get

$$
R_{n}(z, \tau)=-\frac{P_{n}(z, \tau)}{Q_{n}(z, \tau)}=-\frac{\left(c_{n} Z_{n} / Z_{n-1}+\tau\right) \psi_{n-1}(z)}{\left(c_{n} Z_{n} / Z_{n-1}+\tau\right) \phi_{n-1}(z)}=-\frac{\psi_{n-1}(z)}{\phi_{n-1}(z)}
$$

This is independent of $\tau$ for any $\tau \in \hat{\mathbb{C}}$ (not only the real ones) and thus the whole Riemann sphere is mapped to a point, which we denote as $K_{n}(z)$.

We have the following theorem.

Theorem 7.1 Suppose that the index $n$ is regular. Then for $z \in \hat{\mathbb{C}} \backslash \mathbb{T}$

1. The equation of the circle $K_{n}(z)$ is given by

$$
\sum_{k=1}^{n-1}\left|\psi_{k}(z)+s \phi_{k}(z)\right|^{2}+|1-s|^{2}=(s+\bar{s}) \frac{2|z|^{2}}{1-|z|^{2}} .
$$

2. The (closed) disk $\Delta_{n}(z)$ is obtained by replacing the equality sign by $\leq$.

3. The center $c_{n}$ and the radius $r_{n}$ of the disk are

$$
\begin{aligned}
& c_{n}=-\frac{\psi_{n} \bar{\phi}_{n-1}-\psi_{n-1} \bar{\phi}_{n}}{\phi_{n} \bar{\phi}_{n-1}-\phi_{n-1} \bar{\phi}_{n}}=\frac{1}{k_{n-1}(z, z)} \frac{1+|z|^{2}}{1-|z|^{2}} \\
& r_{n}=\left|\frac{\psi_{n} \phi_{n-1}-\psi_{n-1} \phi_{n}}{\phi_{n} \bar{\phi}_{n-1}-\phi_{n-1} \bar{\phi}_{n}}\right|=\frac{1}{k_{n-1}(z, z)}\left|\frac{z}{1-|z|^{2}}\right|
\end{aligned}
$$

where $k_{n-1}(z, z)=\sum_{k=0}^{n-1}\left|\phi_{k}(z)\right|^{2}$.

4. The circle $K_{n}(z)$ is in the right (left) half plane iff $|z|<1 \quad(|z|>1)$.

5. If $m$ is also a regular index and $m>n$, then $\Delta_{m}(z) \subset \Delta_{n}(z)$.

6. The circles $K_{n}(z)$ and $K_{n-1}(z)$ touch.

Proof. We can solve the relation $s=R_{n}(z, \tau)$ for $\tau$ and we then get

$$
\begin{aligned}
\tau & =-\frac{Z_{n-1}(z)}{Z_{n}(z)} \frac{\psi_{n}(z)+s \phi_{n}(z)}{\psi_{n-1}(z)+s \phi_{n-1}(z)} \\
& =-\frac{\left|Z_{n-1}\right|^{2}}{Z_{n} \bar{Z}_{n-1}} \frac{\left[\psi_{n}+s \phi_{n}\right]\left[\overline{\psi_{n-1}+s \phi_{n-1}}\right]}{\left|\psi_{n-1}+s \phi_{n-1}\right|^{2}}
\end{aligned}
$$

Taking the imaginary part gives

$$
\Im \tau=\frac{1}{2 i} \frac{\left|Z_{n-1}\right|^{2}}{\left|\psi_{n-1}+s \phi_{n-1}\right|^{2}} g_{n}(z, s)
$$


with

$$
g_{n}(z, s)=\frac{\left(\overline{\psi_{n}+s \phi_{n}}\right)\left(\psi_{n-1}+s \phi_{n-1}\right)}{\bar{Z}_{n} Z_{n-1}}-\frac{\left(\psi_{n}+s \phi_{n}\right)\left(\overline{\psi_{n-1}+s \phi_{n-1}}\right)}{Z_{n} \bar{Z}_{n-1}}
$$

Now suppose that the index $n$ is regular. Then we can write the equation of the circle $K_{n}(z)$, which corresponds to $\Im \tau=0$ as $g_{n}(z, s)=0$. With the help of Green's formula (5.4) (since $n$ is a regular index and hence $E_{n} \neq 0$ ) we may also write the equation for the circle as

$$
\sum_{k=1}^{n-1}\left|\psi_{k}(z)+s \phi_{k}(z)\right|^{2}+|1-s|^{2}=(s+\bar{s}) \frac{X(z)}{\tilde{H}(z, z)} .
$$

with $X(z)=2 i|z|^{2} /|z-1|^{2}$. This is (1) of the theorem.

Since the denominator $1-|z|^{2}$ is positive, negative or zero, iff $|z|<1,|z|=1$ or $|z|>1$, this means that the circle will be in the right or left half plane depending on $|z|$ being less than 1 or greater than 1 respectively. This is (4).

The disk $\Delta_{n}(z)$ with boundary $K_{n}(z)$ is given by putting a $\leq$ sign instead of equality. Since

$$
(s+\bar{s}) \frac{2|z|^{2}}{1-|z|^{2}}-\sum_{k=1}^{n-1}\left|\psi_{k}(z)+s \phi_{k}(z)\right|^{2}-|1-s|^{2}=A|s|^{2}+B s+\bar{B} \bar{s}+D
$$

with $A=-\sum_{k=0}^{n-1}\left|\phi_{k}\right|^{2}<0$, it follows that this will become negative for $|s|$ sufficiently large. Thus this expression is negative outside the disk. Therefore, the closed disk is described by

$$
\sum_{k=1}^{n-1}\left|\psi_{k}(z)+s \phi_{k}(z)\right|^{2}+|1-s|^{2} \leq(s+\bar{s}) \frac{2|z|^{2}}{1-|z|^{2}}
$$

This is (2).

Since the sum in the left-hand side of (7.1) is non-decreasing with $n$, it follows that we have nested disks for regular indices, i.e., if $m>n$ and $m$ and $n$ regular indices, then $\Delta_{m}(z) \subset \Delta_{n}(z)$. This is (5). We have to exclude here the singular indices because when $n$ is singular, then $\Delta_{n}$ collapses to a point and a subsequent disk with positive radius can not be a subset of $\Delta_{n}$.

Since $R_{n}(z, \infty)=R_{n-1}(z, 0)$, the circles will touch, even if the index $n$ is singular. In the latter case, $K_{n}(z)$ is a point from the circle $K_{n-1}(z)$.

The expressions for center and radius for a general linear fractional transform $(\tau \in \hat{\mathbb{R}})$

$$
s=-\frac{a-\tau b}{c-\tau d}=-\frac{a \bar{d}-b \bar{c}}{c \bar{d}-d \bar{c}}+\frac{a d-b c}{c \bar{d}-d \bar{c}} \cdot \frac{\bar{c}-\tau \bar{d}}{c-\tau d}
$$

are obviously

$$
c=-\frac{a \bar{d}-b \bar{c}}{c \bar{d}-d \bar{c}} \quad \text { and } \quad r=\left|\frac{a d-b c}{c \bar{d}-d \bar{c}}\right| .
$$

Using the Green and Christoffel-Darboux formulas, the expressions for $c_{n}$ and $r_{n}$ as in (3) will follow.

Corollary 7.2 For $z \in\{0, \infty\}$, all the circles $K_{n}(z) n=1,2, \ldots$ reduce to the same point $s(z) \equiv 1$. 
Proof. When $n$ is regular, it follows from the expression for the radius that for $z \in\{0, \infty\}$ it is zero. From the equation of the circle, it follows that $s=1$.

Since successive circles touch also for singular indices, we get the same point for all $n$.

Assume from now on that there are infinitely many regular indices. Because the disks are nested, it follows that when $n(\nu)$ is the sequence of regular indices, then $\Delta_{\infty}=\cap_{n(\nu)} \Delta_{n(\nu)}$ is a disk with radius

$$
r(z)=\lim _{\nu \rightarrow \infty} r_{n(\nu)}(z)
$$

which may reduce to a point when this radius is zero. We have the following

Lemma 7.3 Suppose $z \in \mathbb{C}_{0}=\mathbb{C} \backslash(\mathbb{T} \cup\{0\})$.

If $\Delta_{\infty}(z)$ is a disk (with positive radius), then

$$
\sum_{k=0}^{\infty}\left|\phi_{k}(z)\right|^{2}<\infty \quad \text { and } \quad \sum_{k=0}^{\infty}\left|\psi_{k}(z)\right|^{2}<\infty
$$

If $\Delta_{\infty}(z)$ is a point, then

$$
\sum_{k=0}^{\infty}\left|\phi_{k}(z)\right|^{2}=\infty \quad \text { and } \quad \sum_{k=0}^{\infty}\left|\psi_{k}(z)\right|^{2}=\infty
$$

Proof. It follows from the expression for the radii that $r(z)$ is positive (zero) iff $k_{\infty}(z, z)$ is finite (infinite), i.e., iff $\sum_{k=0}^{\infty}\left|\phi_{k}(z)\right|^{2}$ is finite (infinite).

Let $s$ be a point from the disk $\Delta_{\infty}(z)$. Then it follows from (7.1) that in any case (disk or point)

$$
\sum_{k=1}^{\infty}\left|\psi_{k}(z)+s(z) \phi_{k}(z)\right|^{2}<\infty .
$$

Thus $\sum_{k=1}^{\infty}\left|\phi_{k}(z)\right|^{2}<\infty$ iff $\sum_{k=1}^{\infty}\left|\psi_{k}(z)\right|^{2}<\infty$.

Before we can prove an invariance theorem, we also need the following

Lemma 7.4 If $\phi_{n}$ are the orthonormal functions and $\psi_{n}$ the functions of the second kind, then for any $w \in \mathbb{C}_{0}=\mathbb{C} \backslash(\mathbb{T} \cup\{0\})$

$$
\begin{aligned}
& \phi_{n}(z)=\phi_{n}(w)+\frac{z-w}{\alpha_{n}-z}\left[A_{0 n}+\sum_{k=1}^{n-1} \phi_{k}(z) A_{k n}(w)\right] \\
& \psi_{n}(z)=\psi_{n}(w)+\frac{z-w}{\alpha_{n}-z}\left[B_{0 n}+\sum_{k=1}^{n-1} \psi_{k}(z) A_{k n}(w)\right]
\end{aligned}
$$

where

$$
\begin{gathered}
A_{0 n}(w)=V_{n}(w) \phi_{n}(w)-Y_{n}(w) \psi_{n}(w) \\
B_{0 n}(w)=V_{n}(w) \psi_{n}(w)-Y_{n}(w) \phi_{n}(w), \\
A_{k n}(w)=Y_{n}(w) a_{k n}(w), \quad a_{k n}(w)=\psi_{k}(w) \phi_{n}(w)-\phi_{k}(w) \psi_{n}(w), \quad k=1, \ldots, n-1, \\
Y_{n}(w)=\frac{w-\alpha_{n}}{2 w} \quad \text { and } \quad V_{n}(w)=\frac{w+\alpha_{n}}{2 w} .
\end{gathered}
$$


Proof. From the Christoffel-Darboux formula in Theorem 4.1 and the mixed formula in Theorem 4.2, we get

$$
\begin{aligned}
& \frac{\phi_{n}(z) \phi_{n-1}(w)}{Z_{n}(z) Z_{n-1}(w)}-\frac{\phi_{n}(w) \phi_{n-1}(z)}{Z_{n}(w) Z_{n-1}(z)}=-H(z, w) E_{n} \sum_{k=0}^{n-1} \phi_{k}(z) \phi_{k}(w) \\
& \frac{\phi_{n}(z) \psi_{n-1}(w)}{Z_{n}(z) Z_{n-1}(w)}-\frac{\psi_{n}(w) \phi_{n-1}(z)}{Z_{n}(w) Z_{n-1}(z)}=-H(z, w) E_{n}\left[\sum_{k=1}^{n-1} \phi_{k}(z) \psi_{k}(w)-D(z, w)\right] .
\end{aligned}
$$

Elimination of $\phi_{n-1}(z)$ gives

$$
\frac{\phi_{n}(z)}{Z_{n}(z) Z_{n-1}(w)} a_{n-1, n}(w)=-H(z, w) E_{n}\left[\sum_{k=1}^{n-1} \phi_{k}(z) a_{k, n}(w)-\left[\psi_{n}(w)+D(z, w) \phi_{n}(w)\right]\right]
$$

From the determinant formula of Theorem 4.2, we find

$$
a_{n-1, n}(w)=-E_{n} Z_{n-1}(w) Z_{n}(w) \frac{2 i w}{(1-w)^{2}}
$$

Hence

$$
-\frac{H(z, w) E_{n} Z_{n}(z) Z_{n-1}(w)}{a_{n-1, n}(w)}=\frac{z-w}{\alpha_{n}-z}\left[\frac{w-\alpha_{n}}{2 w}\right]=\frac{z-w}{\alpha_{n}-z} Y_{n}(w)
$$

Thus

$$
\phi_{n}(z)=\frac{z-w}{\alpha_{n}-z} Y_{n}(w)\left[\sum_{k=1}^{n-1} \phi_{k}(z) a_{k n}(w)-\left[\psi_{n}(w)+D(z, w) \phi_{n}(w)\right]\right] .
$$

Next we compute

$$
-\frac{z-w}{\alpha_{n}-z} Y_{n}(w) D(z, w)=\frac{(z+w)\left(\alpha_{n}-w\right)}{2 w\left(\alpha_{n}-z\right)}=1+\frac{(z-w)\left(w+\alpha_{n}\right)}{\left(\alpha_{n}-z\right) 2 w} .
$$

Thus

$$
\begin{aligned}
\phi_{n}(z)=\phi_{n}(w) & +\frac{z-w}{\alpha_{n}-z}\left[V_{n}(w) \phi_{n}(w)-Y_{n}(w) \psi_{n}(w)\right] \\
& +\frac{z-w}{\alpha_{n}-z}\left[\sum_{k=1}^{n-1} \phi_{k}(z)\left[Y_{n}(w) a_{k n}(w)\right]\right]
\end{aligned}
$$

This is the first formula required.

The second formula is proved similarly.

We can now prove the following invariance theorem.

Theorem 7.5 (invariance) Suppose $w \in \mathbb{C}_{0}=\mathbb{C} \backslash(\mathbb{T} \cup\{0\})$ and suppose that $\Delta_{\infty}(w)$ is a disk with positive radius. Then $\Delta_{\infty}(z)$ is a disk with positive radius for every $z \in \mathbb{C}_{0}$ and

$$
\sum_{k=0}^{n}\left|\phi_{k}(z)\right|^{2} \quad \text { and } \quad \sum_{k=0}^{n}\left|\psi_{k}(z)\right|^{2}
$$

converge locally uniformly in $\mathbb{C}_{0}$ as $n \rightarrow \infty$. 
Proof. From Lemma 7.4, we know that with $x_{n}=\phi_{n}$ and $y_{n}=\psi_{n}$ or visa versa, we have, with the notation as in Lemma 7.4

$$
\begin{aligned}
x_{n}(z)=x_{n}(w) & +\frac{z-w}{\alpha_{n}-z} Y_{n}(w) \sum_{k=1}^{n-1} x_{k}(z) a_{k n}(w) \\
& +\frac{z-w}{\alpha_{n}-z}\left[V_{n}(w) x_{n}(w)-Y_{n}(w) y_{n}(w)\right]
\end{aligned}
$$

From the definition of $a_{k n}(w)$, it follows

$$
\begin{aligned}
\sum_{n=1}^{\infty} \sum_{k=1}^{n-1}\left|a_{k n}(w)\right|^{2} & \leq \sum_{n=1}^{\infty} \sum_{k=1}^{n-1}\left(\left|\psi_{k}(w)\right|^{2}\left|\phi_{n}(w)\right|^{2}+\left|\phi_{k}(w)\right|^{2}\left|\psi_{n}(w)\right|^{2}\right) \\
& \leq 2\left(\sum_{n=1}^{\infty}\left|\psi_{n}(w)\right|^{2}\right)\left(\sum_{n=1}^{\infty}\left|\phi_{n}(w)\right|^{2}\right)<\infty
\end{aligned}
$$

by Lemma 7.3. Now suppose thet $C$ is a compact subset of $\mathbb{C}_{0}$. Then for arbitrary $z \in C$ and $w \in \mathbb{C}_{0}$ fixed

$$
\frac{z-w}{\alpha_{n}-z} Y_{n}(w) \text { and } \frac{z-w}{\alpha_{n}-z} V_{n}(w)
$$

are uniformly bounded, say

$$
\left|\frac{z-w}{\alpha_{n}-z} Y_{n}(w)\right| \leq R_{1} \quad \text { and } \quad\left|\frac{z-w}{\alpha_{n}-z} V_{n}(w)\right| \leq R_{2}
$$

So

$$
\begin{aligned}
\left(\sum_{n=m}^{N}\left|x_{n}(z)\right|^{2}\right)^{1 / 2} \leq & \left(\sum_{n=m}^{N}\left|x_{n}(w)\right|^{2}\right)^{1 / 2}+R_{1}\left(\sum_{n=m}^{N}\left|y_{n}(w)\right|^{2}\right)^{1 / 2} \\
& +R_{2}\left(\sum_{n=m}^{N}\left|x_{n}(w)\right|^{2}\right)^{1 / 2}+R_{1}\left(\sum_{n=m}^{N}\left|\sum_{k=1}^{n-1} a_{k n}(w) x_{k}(z)\right|^{2}\right)^{1 / 2}
\end{aligned}
$$

For any $0<\epsilon<1$, choose $1<m=m\left(\epsilon, R_{1}, R_{2}\right)$ such that

$$
\left(\sum_{n=m}^{\infty}\left|y_{n}(w)\right|^{2}\right)^{1 / 2}<\frac{\epsilon}{R_{1}}, \quad\left(\sum_{n=m}^{\infty}\left|x_{n}(w)\right|^{2}\right)^{1 / 2}<\frac{\epsilon}{1+R_{2}}
$$

and

$$
\left(\sum_{n=m}^{\infty} \sum_{k=1}^{n-1}\left|a_{k n}(w)\right|^{2}\right)^{1 / 2}<\frac{\epsilon}{R_{1}}
$$

Then

$$
\begin{aligned}
\left(\sum_{n=m}^{N}\left|x_{n}(z)\right|^{2}\right)^{1 / 2} & \leq \epsilon+\epsilon+R_{1}\left[\sum_{n=m}^{N}\left(\sum_{k=1}^{n-1}\left|a_{k n}(w)\right|^{2}\right)\left(\sum_{k=1}^{n-1}\left|x_{k}(z)\right|^{2}\right)\right]^{1 / 2} \\
& \leq 2 \epsilon+R_{1}\left(\sum_{k=1}^{N}\left|x_{k}(z)\right|^{2}\right)^{1 / 2}\left(\sum_{n=m}^{\infty} \sum_{k=1}^{n-1}\left|a_{k n}(w)\right|^{2}\right)^{1 / 2} \\
& \leq 2 \epsilon+\epsilon\left(\sum_{k=1}^{N}\left|x_{k}(z)\right|^{2}\right)^{1 / 2} \\
& \leq 2 \epsilon+\epsilon\left(\sum_{k=m}^{N}\left|x_{k}(z)\right|^{2}\right)^{1 / 2}+\epsilon\left(\sum_{k=1}^{m-1}\left|x_{k}(z)\right|^{2}\right)^{1 / 2}
\end{aligned}
$$


Hence

$$
(1-\epsilon)\left(\sum_{n=m}^{N}\left|x_{n}(z)\right|^{2}\right)^{1 / 2} \leq 2 \epsilon+\epsilon\left(\sum_{k=1}^{m-1}\left|x_{k}(z)\right|^{2}\right)^{1 / 2}
$$

Because $\sum_{k=1}^{m-1}\left|x_{k}(z)\right|^{2}$ is a continuous function in $C$, there is an $M>0$ such that

$$
\left(\sum_{k=1}^{m-1}\left|x_{k}(z)\right|^{2}\right)^{1 / 2} \leq M
$$

holds uniformly in $C$. Thus

$$
\left(\sum_{k=1}^{N}\left|x_{k}(z)\right|^{2}\right)^{1 / 2} \leq \frac{(2+M) \epsilon}{1-\epsilon}, \quad N \geq m
$$

This proves the uniform convergence of $\sum_{n=0}^{\infty}\left|x_{k}(z)\right|^{2}$ in $C$.

We can now also prove the analyticity theorem.

Theorem 7.6 (analyticity) Let $\Delta_{\infty}(z)$ be a point. Then the limit

$$
s(z)=\lim _{n \rightarrow \infty} R_{n}(z, \tau), \quad z \in \mathbb{C} \backslash \mathbb{T}
$$

is an analytic function of $z$ not depending on $\tau$. Moreover

$$
\frac{\Re s(z)}{1-|z|^{2}}>0, \quad z \in \mathbb{C} \backslash \mathbb{T}
$$

Proof. By Theorem $7.1(6)$, it follows that if $\Delta_{\infty}(z)$ is a point then

$$
\lim _{n \rightarrow \infty} R_{n}(z, \tau)=\lim _{n \rightarrow \infty} s_{n}(z)=s(z)
$$

exists and is independent of $\tau$.

Obviously $s_{n}(z)=R_{n}(z, \tau)$ is analytic for $z \in \mathbb{C} \backslash \mathbb{T}$. Thus the analyticity of $s(z)$ will follow if the functions $s_{n}(z)$ are uniformly bounded in compact subsets of $\mathbb{C} \backslash \mathbb{T}$. This is shown as follows. We know that

$$
\left|1-s_{n}(z)\right|^{2}+\sum_{k=1}^{n-1}\left|\psi_{k}(z)+s_{n}(z) \phi_{n}(z)\right|^{2}=\left(s_{n}(z)+\bar{s}_{n}(z)\right) \frac{2|z|^{2}}{1-|z|^{2}} \text {. }
$$

Thus

$$
1+\left|s_{n}\right|^{2}+\sum_{k=1}^{n-1}\left|\psi_{k}+s_{n} \phi_{n}\right|^{2}=\left(s_{n}+\bar{s}_{n}\right) \frac{1+|z|^{2}}{1-|z|^{2}}
$$

or, using $\left|s_{n}+\bar{s}_{n}\right| \leq 2\left|s_{n}\right|$,

$$
\left|s_{n}\right|^{2}<1+\left|s_{n}\right|^{2}+\sum_{k=1}^{n-1}\left|\psi_{k}+s_{n} \phi_{n}\right|^{2} \leq 2\left|s_{n}\right|\left|\frac{1+|z|^{2}}{1-|z|^{2}}\right| .
$$

Therefore

$$
\left|s_{n}(z)\right| \leq\left|\frac{1+|z|^{2}}{1-|z|^{2}}\right| .
$$

Since the right-hand side is uniformly bounded in compact subsets of $\mathbb{C} \backslash \mathbb{T}$, the analyticity of $s(z)$ follows.

The last inequality is a direct consequence of Theorem 7.1 (4). 


\section{The moment problem}

We are now ready to border the moment problem as it was described in the introduction. Thus given the linear functional $M$, real and positive on $\mathcal{R}$, can we find the measure $\mu$, finite and positive on $[-\pi, \pi]$, such that

$$
M\left\{\frac{1}{\omega_{n}}\right\}=\mu_{n}=\int_{-\pi}^{\pi} \frac{d \mu(\theta)}{\omega_{n}\left(e^{i \theta}\right)}, \quad n=0,1, \ldots
$$

Note that if $\mu$ is a solution of this moment problem, then it is easily seen that $M\{f\}=$ $\int_{-\pi}^{\pi} f\left(e^{i \theta}\right) d \mu(\theta)$ for all $f \in \mathcal{L}$. Thus $\mu$ defines the linear functional $M$ in the space $\mathcal{L}$. The given functional $M$ was however defined on the larger space $\mathcal{R}=\mathcal{L} \cdot \mathcal{L}$. Thus strictly speaking, to solve the moment problem, it would have been sufficient to define $M$ on $\mathcal{L}$ only. As we have seen however, our approach so far relied heavily on orthogonality properties, i.e., on the fact that we have a real and positive inner product, and that is why we needed the functional $M$ to be defined on $\mathcal{R}$.

Another, more general moment problem which could have been considered, is to write the functional $M$ as an integral with a positive measure $\mu$ for all functions in $\mathcal{R}=\mathcal{L} \cdot \mathcal{L}$ (and not just for functions in $\mathcal{L}$ ). Thus $M\{f\}=\int_{-\pi}^{\pi} f\left(e^{i \theta}\right) d \mu(\theta)$ for all $f \in \mathcal{R}$. Then we should consider the problem of finding a positive measure $\mu$ such that the following moment relations

$$
M\left\{\frac{1}{\omega_{n} \omega_{m *}}\right\}=\mu_{n m}=\int_{-\pi}^{\pi} \frac{d \mu(\theta)}{\omega_{n}\left(e^{i \theta}\right) \omega_{m *}\left(e^{i \theta}\right)}, \quad n, m=0,1, \ldots
$$

hold. This moment problem is indeed equivalent to finding a positive measure $\mu$ such that

$$
\langle f, g\rangle=M\left\{f g_{*}\right\}=\int_{-\pi}^{\pi} f\left(e^{i \theta}\right) \overline{g\left(e^{i \theta}\right)} d \mu(\theta), \quad \forall f, g \in \mathcal{L} .
$$

We shall call this the moment problem in $\mathcal{R}=\mathcal{L} \cdot \mathcal{L}$, while the previously explained simpler form is called the moment problem in $\mathcal{L}$. Note that the moment problem in $\mathcal{R}$ prescribes also the moments $\mu_{n 0}=\mu_{n}$, which implies that a solution of the moment problem in $\mathcal{R}$ will automatically be a solution of the moment problem in $\mathcal{L}$. It is not clear whether the converse is true in general.

It was shown in [3] that the moment problem in $\mathcal{L}$ has at least one solution. Since the proof of [3] is rather compact, we include it here in more detail. The arguments which are made explicit in this proof also show why the same arguments do not lead to a solution of the moment problem in $\mathcal{R}$.

Theorem 8.1 Let $M$ be a real positive functional defined on $\mathcal{R}=\mathcal{L} \cdot \mathcal{L}$ and assume that the sequence of orthogonal rational functions $\phi_{n}$ has infinitely many regular indices. Then there exists at least one measure $\mu$ on $\mathbb{T}$ which solves the moment problem in $\mathcal{L}$, i.e., which satisfies (8.1).

Proof. The proof is based on the fact that the discrete measures $\mu_{k}$ representing the quadrature formulas

$$
\sum_{i=1}^{k} \lambda_{k i}(\tau) f\left(\xi_{k i}(\tau)\right)=\int_{-\pi}^{\pi} f\left(e^{i \theta}\right) d \mu_{k}(\theta)
$$

which are exact in $\mathcal{R}_{k-1}$, hence also in $\mathcal{L}_{k-1}$, are uniformly bounded by $M\{1\}=1$ and hence by Helly's selection principle, the sequence $\left\{\mu_{k}\right\}$ will have a convergent subsequence 
$\mu_{k(j)} \rightarrow \mu$. Next we prove that such a $\mu$ solves the moment problem in $\mathcal{L}$, i.e., that

$$
M\left\{\frac{1}{\omega_{n}}\right\}=\int_{-\pi}^{\pi} \frac{d \mu(\theta)}{\omega_{n}\left(e^{i \theta}\right)}, \quad n=0,1, \ldots
$$

For $n=0$, we can apply Helly's convergence theorem since 1 is a continuous function in the interval $[-\pi, \pi]$. Thus it remains to prove this for a fixed $n>0$. We denote the elements of the subsequence $k(j)$ by $k$ for simplicity. Note that for $n>0$ we can not simply apply Helly's convergence theorem because the $\omega_{n}^{-1}$ are not continuous on the interval $I=[-\pi, \pi]$. However, consider any subset $J \subset I$ which does not contain the $\alpha_{1}, \ldots, \alpha_{n}$, then $\omega_{n}^{-1}$ is indeed continuous on $J$ and this means that we can find a $k=k(j)$ large enough such that for a given $\epsilon>0$,

$$
\left|\int_{J} \frac{d \mu(\theta)-d \mu_{k}(\theta)}{\omega_{n}\left(e^{i \theta}\right)}\right|<\frac{\epsilon}{3}
$$

On the other hand, for $k=k(j)>n$,

$$
\begin{aligned}
\left|\int_{I-J} \frac{d \mu_{k}(\theta)}{\omega_{n}\left(e^{i \theta}\right)}\right| & =\left|\int_{I-J} \frac{\omega_{n *}\left(e^{i \theta}\right) d \mu_{k}(\theta)}{\left|\omega_{n}\left(e^{i \theta}\right)\right|^{2}}\right| \\
& \leq \max _{I-J}\left|\omega_{n *}\left(e^{i \theta}\right)\right| \int_{I-J} \frac{d \mu_{k}(\theta)}{\left|\omega_{n}\left(e^{i \theta}\right)\right|^{2}} \leq \max _{I-J}\left|\omega_{n *}\left(e^{i \theta}\right)\right| M\left\{\frac{1}{\omega_{n} \omega_{n *}}\right\} .
\end{aligned}
$$

We can always choose $J$ large enough such that the maximum of $\left|\omega_{n *}\right|$ in $I-J$ is arbitrary small. Because $M\left\{\left[\omega_{n} \omega_{n *}\right]^{-1}\right\}$ is finite, it follows that for any $\epsilon>0$, we can make $J$ large enough to satisfy

$$
\left|\int_{I-J} \frac{d \mu_{k}(\theta)}{\omega_{n}\left(e^{i \theta}\right)}\right|<\frac{\epsilon}{3} .
$$

Note that this holds for any $k>n$, i.e., $J$ is independent of $k$. Next we want to show that $J$ can also be made large enough to satisfy

$$
\left|\int_{I-J} \frac{d \mu(\theta)}{\omega_{n}\left(e^{i \theta}\right)}\right|<\frac{\epsilon}{3}
$$

To obtain this, we consider sets $J_{p}$ none of which contain $\alpha_{1}, \ldots, \alpha_{n}$ and such that $J_{q} \subset J_{p}$ for $p>q$ and $\cup_{p} J_{p}=I$. Note that for $k=k(j)>n$ and for any $p$

$$
\int_{J_{p}} \frac{d \mu(\theta)}{\omega_{n}\left(e^{i \theta}\right)}=\int_{J_{p}} \frac{d \mu(\theta)-d \mu_{k}(\theta)}{\omega_{n}\left(e^{i \theta}\right)}+\int_{J_{p}} \frac{d \mu_{k}(\theta)}{\omega_{n}\left(e^{i \theta}\right)} .
$$

Thus if $p>q$ and $k>n$

$$
\left|\int_{J_{p}-J_{q}} \frac{d \mu(\theta)}{\omega_{n}\left(e^{i \theta}\right)}\right| \leq\left|\int_{J_{p}-J_{q}} \frac{d \mu(\theta)-d \mu_{k}(\theta)}{\omega_{n}\left(e^{i \theta}\right)}\right|+\left|\int_{J_{p}-J_{q}} \frac{d \mu_{k}(\theta)}{\omega_{n}\left(e^{i \theta}\right)}\right| .
$$

By (8.4), there is always a $p$ and $q$ large enough such that for any $\eta>0$

$$
\left|\int_{J_{p}-J_{q}} \frac{d \mu_{k}(\theta)}{\omega_{n}\left(e^{i \theta}\right)}\right|<\frac{\eta}{2}
$$


for all large $k$. By (8.3), we can make $k$ so large that for any $\eta>0$

$$
\left|\int_{J_{p}-J_{q}} \frac{d \mu(\theta)-d \mu_{k}(\theta)}{\omega_{n}\left(e^{i \theta}\right)}\right|<\frac{\eta}{2}
$$

Combining (8.6), (8.7) and (8.8) shows that it is possible to make

$$
\left|\int_{J_{p}} \frac{d \mu(\theta)}{\omega_{n}\left(e^{i \theta}\right)}-\int_{J_{q}} \frac{d \mu(\theta)}{\omega_{n}\left(e^{i \theta}\right)}\right|<\eta
$$

for any $\eta>0$. This means that

$$
\left\{\int_{J_{p}} \frac{d \mu(\theta)}{\omega\left(e^{i \theta}\right)}\right\}_{p}
$$

is a Cauchy sequence so that its limit for $p \rightarrow \infty$ (which is the integral over $I$ ) exists, thus that for any $\epsilon>0$ there exists a $p$ large enough such that

$$
\left|\int_{J_{p}} \frac{d \mu(\theta)}{\omega_{n}\left(e^{i \theta}\right)}-\int_{I} \frac{d \mu(\theta)}{\omega_{n}\left(e^{i \theta}\right)}\right|<\frac{\epsilon}{3}
$$

which proves (8.5). Finally, by (8.4), (8.5) and (8.3),

$$
\left|\int_{I} \frac{d \mu_{k}(\theta)-d \mu(\theta)}{\omega_{n}\left(e^{i \theta}\right)}\right| \leq\left|\int_{I-J} \frac{d \mu_{k}(\theta)}{\omega_{n}\left(e^{i \theta}\right)}\right|+\left|\int_{I-J} \frac{d \mu(\theta)}{\omega_{n}\left(e^{i \theta}\right)}\right|+\left|\int_{J} \frac{d \mu_{k}(\theta)-d \mu(\theta)}{\omega_{n}\left(e^{i \theta}\right)}\right|<\epsilon
$$

because each of the terms in the right-hand side can be bounded by $\epsilon / 3$. Thus

$$
\lim _{k(j) \rightarrow \infty} \int_{I} \frac{d \mu_{k(j)}(\theta)}{\omega_{n}\left(e^{i \theta}\right)}=M\left\{\frac{1}{\omega_{n}}\right\}=\int_{I} \frac{d \mu(\theta)}{\omega_{n}\left(e^{i \theta}\right)} .
$$

This proves the theorem.

We now use our framework of nested disks to obtain information about when the solution is unique. Let us denote by $\mathcal{M}^{\mathcal{L}}$ the set of solutions of the moment problem in $\mathcal{L}$ and $\mathcal{M}^{\mathcal{R}}$ the set of solutions of the moment problem in $\mathcal{R}$. Then we have

Theorem 8.2 Assume that the sequence of orthonormal functions $\phi_{n}$ has infinitely many regular indices. Hence $\mathcal{M}^{\mathcal{L}} \neq \varnothing$. Fix $z \in \mathbb{C} \backslash \mathbb{T}$ and define for $\mu \in \mathcal{M}^{\mathcal{L}}$ its Riesz-Herglotz transform

$$
\Omega_{\mu}(z)=\int_{-\pi}^{\pi} D\left(e^{i \theta}, z\right) d \mu(\theta)
$$

Then

$$
\left\{\Omega_{\mu}(z): \mu \in \mathcal{M}^{\mathcal{R}}\right\} \subset \Delta_{\infty}(z) \subset\left\{\Omega_{\mu}(z): \mu \in \mathcal{M}^{\mathcal{L}}\right\} .
$$

Proof. Let $s=\Omega_{\mu}(z)$ for some $\mu \in \mathcal{M}^{\mathcal{R}}$. Note that the system $\left\{\phi_{n}\right\}$ is then orthonormal with respect to the inner product defined by the measure $\mu$. Let $f(z)=\overline{D(t, z)}, t \in \mathbb{T}$. Writing the generalized Fourier series of $f(z)$ as

$$
f(z) \sim \sum_{k=0}^{\infty} \gamma_{k} \phi_{k}(z), \quad \gamma_{k}=\left\langle f, \phi_{k}\right\rangle,
$$


then using

$$
\gamma_{k}=\int_{-\pi}^{\pi} \overline{D(t, z) \phi_{k}(t)} d \mu(\theta)
$$

it turns out that $\bar{\gamma}_{0}=s$ and $\bar{\gamma}_{k}=\psi_{k}(z)+s \phi_{k}(z)$ for $k \geq 1$ because

$$
\bar{\gamma}_{k}=\int_{-\pi}^{\pi} D(t, z) \phi_{k}(t) d \mu(\theta)=\int_{-\pi}^{\pi} D(t, z)\left[\phi_{k}(t)-\phi_{k}(z)\right] d \mu(\theta)+\phi_{k}(z) \Omega_{\mu}(z), \quad t=e^{i \theta} .
$$

On the other hand, it can be shown that for $t \in \mathbb{T}$

$$
-1+\frac{1+|z|^{2}}{1-|z|^{2}}[D(t, z)+\overline{D(t, z)}]=\frac{|t+z|^{2}}{|t-z|^{2}}=|D(t, z)|^{2} .
$$

Using Bessel's inequality, it then follows that

$$
\sum_{k=0}^{\infty}\left|\gamma_{k}\right|^{2}=|s|^{2}+\sum_{k=1}^{\infty}\left|\psi_{k}+s \phi_{k}\right|^{2} \leq-1+\frac{1+|z|^{2}}{1-|z|^{2}}(s+\bar{s})
$$

which can be rearranged as

$$
|1-s|^{2}+\sum_{k=1}^{\infty}\left|\psi_{k}+s \phi_{k}\right|^{2} \leq \frac{2|z|^{2}}{1-|z|^{2}}(s+\bar{s})
$$

This means that $s \in \Delta_{\infty}(z)$.

Next it is shown that if $s \in \Delta_{\infty}(z)$, then it is the Riesz-Herglotz transform of some $\mu \in \mathcal{M}^{\mathcal{L}}$. This is readily shown by using the quadrature formulas we have discussed. We consider the limiting point and the limiting disk case separately.

If $\Delta_{\infty}(z)$ is a point, then since $s \in \Delta_{\infty}(z)$, there must exist $s_{n} \in K_{n}(z)$ such that $s_{n} \rightarrow s$. Since there is for each $n$ some $\tau_{n}$ such that

$$
s_{n}=R_{n}\left(z, \tau_{n}\right)=\int_{-\pi}^{\pi} D\left(e^{i \theta}, z\right) d \mu_{n}(\theta) .
$$

Helly's selection criterion then yields that there is a subsequence $\mu_{n(j)} \rightarrow \mu$. By the proof of the previous theorem, $\mu \in \mathcal{M}^{\mathcal{L}}$ and by Helly's convergence theorem

$$
\lim _{j \rightarrow \infty} \int_{-\pi}^{\pi} D(t, z) d \mu_{n(j)}(\theta)=\int_{-\pi}^{\pi} D(t, z) d \mu(\theta)=s .
$$

Thus $s$ is the Riesz-Herglotz transform of a $\mu \in \mathcal{M}^{\mathcal{L}}$.

If $\Delta_{\infty}(z)$ is a disk, let $s$ be a point on the boundary $K_{\infty}(z)$. Recall that for a fixed $n$, we can, except for finitely many values of $\tau$, associate a quadrature formula with $R_{n}(z, \tau)$. Let us denote the discrete measure that is associated with this quadrature by $\mu_{n}(\cdot, \tau)$. It depends on $n$ but also on the choice of $\tau$. We can then, for every regular index $n$ choose an $s_{n} \in K_{n}(z)$ such that these $s_{n}$ tend to $s$ and such that $s_{n}=\Omega_{\mu_{n}}(z)$, where $\mu_{n}=\mu_{n}\left(\cdot, \tau_{n}\right)$ and where $\tau_{n}$ is chosen such that $s_{n}=R_{n}\left(z, \tau_{n}\right)$. By Helly's theorems and the proof of the previous theorem, there exists a $\mu \in \mathcal{M}^{\mathcal{L}}$ such that $\Omega_{\mu}(z)=s$.

Thus every $s$ on the boundary $K_{\infty}(z)$ is of the form $\Omega_{\mu}(z)$ with $\mu \in \mathcal{M}^{\mathcal{L}}$. Now let $s$ be an interior point of $\Delta_{\infty}(z)$. Then it can be found as a convex combination $s=\lambda s_{1}+(1-\lambda) s_{2}$ $(0<\lambda<1)$ of points $s_{1}, s_{2}$ on the boundary $K_{\infty}(z)$. Thus there exist $\mu_{1}, \mu_{2} \in \mathcal{M}^{\mathcal{L}}$ such that

$$
s_{j}=\int_{-\pi}^{\pi} D(t, z) d \mu_{j}(\theta), \quad t=e^{i \theta} .
$$

Thus $\mu=\lambda \mu_{1}+(1-\lambda) \mu_{2} \in \mathcal{M}^{\mathcal{L}}$ and $s=\Omega_{\mu}(z)$.

Now the following corollary is obvious. 
Corollary 8.3 In the case of a limiting disk, for each $s \in \Delta_{\infty}(z), z \in \mathbb{C}_{0}=\mathbb{C} \backslash(\mathbb{T} \cup\{0\})$, there are infinitely many $\mu \in \mathcal{M}^{\mathcal{L}}$ such that $s=\Omega_{\mu}(z)$. The moment problem in $\mathcal{L}$ has infinitely many solutions.

In the case of a limiting point, a solution of the moment problem in $\mathcal{R}$ is unique.

\section{References}

[1] N.I. Akhiezer. The classical moment problem. Oliver and Boyd, Edinburgh, 1969. Originally published Moscow, 1961.

[2] A. Bultheel and P. Dewilde. Orthogonal functions related to the Nevanlinna-Pick problem. In P. Dewilde, editor, Proc. 4th Int. Conf. on Math. Theory of Networks and Systems at Delft, pages 207-212, North-Hollywood, 1979. Western Periodicals.

[3] A. Bultheel, P. González-Vera, E. Hendriksen, and O. Njåstad. Orthogonal rational functions with poles on the unit circle. J. Math. Anal. Appl., 182:221-243, 1994.

[4] A. Bultheel, P. González-Vera, E. Hendriksen, and O. Njåstad. Orthogonal rational functions and nested disks. J. Approx. Theory, 1994. Accepted.

[5] A. Bultheel, P. González-Vera, E. Hendriksen, and O. Njåstad. Orthogonality and boundary interpolation. In A.M. Cuyt, editor, Nonlinear Numerical Methods and Rational Approximation II, pages 37-48. Kluwer, 1994.

[6] A. Bultheel, P. González-Vera, E. Hendriksen, and O. Njåstad. Recurrence relations for orthogonal functions. In S.C. Cooper and W.J. Thron, editors, Continued Fractions and Orthogonal Functions, volume 154 of Lecture Notes in Pure and Appl. Math., pages 24-46. Marcel Dekker, 1994.

[7] A. Bultheel, P. González-Vera, E. Hendriksen, and O. Njåstad. Orthogonal rational functions and modified approximants. Numer. Algorithms, 11:57-69, 1996.

[8] P. Dewilde, A. Viera, and T. Kailath. On a generalized szegö-Levinson realization algorithm for optimal linear predictors based on a network synthesis approach. IEEE Trans. Circuits and Systems, CAS-25:663-675, 1978.

[9] M.M. Djrbashian. A survey on the theory of orthogonal systems and some open problems. In P. Nevai, editor, Orthogonal polynomials: Theory and practice, volume 294 of Series C: Mathematical and Physical Sciences, pages 135-146, Boston, 1990. NATOASI, Kluwer Academic Publishers.

[10] H. Hamburger. Ueber eine Erweiterung des Stieltjesschen Moment Problems I. Math. Ann., 81:235-319, 1920.

[11] H. Hamburger. Ueber eine Erweiterung des Stieltjesschen Moment Problems II. Math. Ann., 82:120-164, 1921.

[12] H. Hamburger. Ueber eine Erweiterung des Stieltjesschen Moment Problems II. Math. Ann., 82:168-187, 1921. 
[13] W.B. Jones, O. Njåstad, and W.J. Thron. Orthogonal Laurent polynomials and the strong Hamburger moment problem. J. Math. Anal. Appl., 98:528-554, 1984.

[14] H.J. Landau, editor. Moments in mathematics, volume 37 of Proc. Sympos. Appl. Math. Amer. Math. Soc., Providence, R.I., 1987.

[15] O. Njåstad. An extended Hamburger moment problem. Proc. Edinburgh Math. Soc., 28:167-183, 1985.

[16] O. Njåstad. Unique solvability of an extended Hamburger moment problem. J. Math . Anal. Appl., 124:502-519, 1987.

[17] O. Njåstad and W.J. Thron. Unique solvability of the strong Hamburger moment problem. J. Austral. Math. Soc. (Series A), 40:5-19, 1986.

[18] A.A. Nudel'man. Some properties of linear fractional transformations and the harmonic mean of matrices. In I. Gohberg and L.A. Sakhnovich, editors, Matrix and operator valued functions, volume 72 of Oper. Theory: Adv. Appl., pages 171-184. Birkhäuser Verlag, 1994.

[19] A.A. Nudelmann. Multipoint matrix moment problem. Dokl. Acad. Nauk., 298:812-815, 1988.

[20] J.A. Shohat and J.D. Tamarkin. The problem of moments, volume 1 of Math. Surveys. Amer. Math. Soc., Providence, R.I., 1943.

[21] T.J. Stieltjes. Recherches sur les fractions continues. Ann. Fac. Sci. Toulouse, 8:J.1122, 9:1-47, 1894. Enlish transl.: Oeuvres Complèts, Collected Papers, Springer Verlag, 1993, Vol. 2, 609-745.

[22] M.H. Stone. Linear transformations in Hilbert space and their applications to analysis, volume 15 of American Math. Soc. Coll. Publ. Amer. Math. Soc., New York, 1932.

[23] H. Weyl. Ueber gewöhnliche Differentialgeleichungen mit Singularitäten und die Zugehörigen Entwicklungen willkürlicher Funktionen. Math. Ann., 68:220-269, 1910. 